\title{
Light-front Ward-Takahashi identity and current conservation
}

\author{
J. A. O. Marinho, ${ }^{1}$ T. Frederico, ${ }^{1}$ and P. U. Sauer ${ }^{2}$ \\ ${ }^{1}$ Instituto Tecnológico de Aeronáutica, 12.228-900 São José dos Campos, SP, Brazil \\ ${ }^{2}$ Institute for Theoretical Physics, University Hannover, D-30167 Hannover, Germany
}

(Received 2 February 2007; revised manuscript received 16 July 2007; published 1 November 2007)

\begin{abstract}
We construct a conserved electromagnetic current for two-boson systems in finite mass boson exchange models within light-front dynamics. For that purpose, we use a quasipotential reduction to perform the three-dimensional light-front projection of the 4-dimensional current operator. The electromagnetic current operator acting on the valence component of bound and scattering states can be perturbatively calculated in the quasipotential expansion, in correspondence with a truncation of the Fock space. The divergence of the proposed current operator satisfies a Ward-Takahashi identity at any given order of the quasipotential expansion. Also shown is the relation between the three-dimensional light-front reduction of the field-theoretic Bethe-Salpeter amplitudes and current for bound and scattering states with the 4dimensional ones. The matrix elements of the 4-dimensional current operator can be fully recovered from the corresponding light-front ones. In one test case the theoretical framework is realized explicitly.
\end{abstract}

DOI: 10.1103/PhysRevD.76.096001

PACS numbers: 11.10.St, 11.40.- q, 13.40.-f, 21.45.+v

\section{MOTIVATION}

The dynamics of an interacting quantum system can be fully described by the evolution between space-time hyperplanes which correspond to given global times. The hyperplanes $x^{0}=$ const define the instant-form dynamics and the hyperplanes $x^{+}=x^{0}+x^{3}=$ const the light-front (LF) dynamics [1]. The particular light-front hyperplane, defined by the global time $x^{+}=0$, is left invariant by seven kinematical Poincaré generators, while the other hyperplanes $x^{+}=$const scale under light-front boosts. In contrast, the covariant formulation of field theory relies on the individual times $x_{i}^{0}$ or on the individual light-front times $x_{i}^{+}$.

One possible covariant approach to study interacting systems is through the Bethe-Salpeter equation [2]. Its solution provides 4-dimensional amplitudes that together with observable operators allow one to calculate matrix elements related to physical processes [3].

In the light-front quantization of field theory [4], the wave function of the interacting system at $x^{+}=0$ is an eigenstate of the Hamiltonian $P^{-}$, and matrix elements of observable operators defined at $x^{+}=0$ are calculated to yield physical quantities. The wave function of a composite system spreads out in all Fock sectors having the quantum numbers of the physical state. One possibility to link the Bethe-Salpeter (BS) formalism and light-front quantization is through the quasipotential (QP) approach to the BS equation [5], as implemented by the works of Sales et al. [6,7].

The three-dimensional reduction of the Bethe-Salpeter equation performed in the framework of the quasipotential approach to light-front dynamics allows one to calculate within a systematic expansion the effective interaction acting on the valence component of the wave function. The effective interaction can be calculated perturbatively and it is in correspondence with an expansion of the composite system wave function in the Fock space. From the 4-dimensional definition of an observable operator, the light-front counterpart can be as well calculated perturbatively within the quasipotential expansion. In particular, the three-dimensional or light-front current operator can be derived accordingly [6,7].

A problem arises when the quasipotential is truncated, and the light-front current operator is calculated with truncated quantities. The current is not anymore conserved and it violates the Ward-Takahashi identity (WTI), as the expansion of the effective interaction and current are not consistently matched. Recently, Kvinikhidze and Blankleider [8] by using gauging techniques were able to obtain a current that satisfies a WTI, and therefore is conserved. An earlier perturbative approach to the WTI within light-front quantization was performed in Ref. [9].

The aim of this work is to propose a solution to the problem of nonconservation of the electromagnetic current in a truncated light-front reduction of the 4-dimensional current operator for an interacting two-boson system in finite mass boson exchange models within the quasipotential framework. The paper demonstrates that, while the truncation of the expansion of the current and effective interaction violates covariance, current conservation can be restored by a proper choice of the truncation in the expansion of the current operator. Our framework allows one to treat at the same level the current operator for bound and scattering states. The derived current operator and its truncation applies to elastic and inelastic processes, as well as to radiative photon emission in the inelastic scattering of two particles.

The technical procedure is developed for a two-boson case and the construction of the current is straightforward. Our framework goes beyond the ladder approximation; it allows one to include as well an interacting part in the 4dimensional current, e.g., crossed exchanges or charged exchanges, if the corresponding solution of the light-front 
Bethe-Salpeter equation is aimed. The step from the threedimensional light-front reduction of the field-theoretic Bethe-Salpeter amplitudes and currents to the 4dimensional representation is also discussed. The matrix elements of the 4-dimensional current operator can be fully recovered from the corresponding light-front ones.

The formal procedure is realized in practice for the ladder Bethe-Salpeter equation in one test case. We computed all Lorentz components of the current operator in the lowest nontrivial order of the quasipotential expansion. This is necessary in order to obtain explicitly the WardTakahashi identity, which we found in agreement with the expression derived formally. In the example, the good $(+)$, transverse, and bad $(-)$ components of the current operator are calculated in the one-boson-exchange approximation. The reference frame is kept quite general to study different possibilities of plus momentum transfers $Q^{+} \geq 0$. For non-Drell-Yan-West frames the pair contribution to the current $[10,11]$ is evaluated, as also required by current conservation. Moreover, the pair contribution to the bad current in the limit of $Q^{+} \rightarrow 0$ is analyzed in more detail to point out that no singular behavior is found in the test case, in contrast to models that have pointlike vertices [12]. One should note that, when the mass $\mu$ of the exchanged boson and the coupling constant $g_{S}$ are taken to infinity while the ratio $g_{S} / \mu$ is kept constant, the model is reduced to a model with pointlike vertices. However, the limits of $Q^{+} \rightarrow 0$ and $\mu \rightarrow \infty$ with finite $g_{S} / \mu$ do not commute in the evaluation of the bad component of the current and the end-point singularity still remains with pointlike vertices. The example is closed with a brief discussion of current conservation and the issue of full covariance.

The study of composite hadronic systems with electromagnetic probes makes the formulation of the conserved vector current operator, to be used together with valence light-front wave functions, particularly useful. For example, in phenomenological model studies of meson electromagnetic form factors in a non-Drell-Yan-West frame, e.g. in the Lev-Pace-Salmè frame $Q^{+}>0$ [13], it was shown that a pair current term is necessary to keep covariance and current conservation as seen in simple analytical models $[10,11]$.

The gauge invariant method of Gross and Riska [14], and the related method of gauging equations of Kvinikhidze and Blankleider [15], was used by Tiburzi and Miller [16] to construct the light-front electromagnetic current for bound states in lowest order of the interaction. Their framework was applied to investigate the electromagnetic properties of the bound state in scalar models. They compute the elastic form factor in $1+1$ dimensions for $Q^{+}>0$ frames, the timelike form factor, and generalized parton distribution in $3+1$ dimensions. The current operator implied in contributions of non-wave-function vertices to electromagnetic observables, originated by the production of a particle-antiparticle pair by the virtual photon with $Q^{+}>0$.

Also, mesonic decays demand non-Drell-Yan-West frames [17] as well as timelike form factors [18-20]. An expansion of the light-front current operator that allows current conservation could have an impact on phenomenological analysis of electromagnetic elastic and inelastic process of QCD based processes [4]. Our work provides the missing link between the valence wave function in finite mass boson exchange models and the corresponding conserved current operator for elastic and inelastic processes in a truncated Fock space.

The outline of the work is the following. In Sec. II, we review the quasipotential approach to light-front dynamics, the Bethe-Salpeter formalism, the electromagnetic charge and current operators, and the 4-dimensional operator form of the Ward-Takahashi identity (WTI), that leads to current conservation. Furthermore, we look again over the projection on the light-front time and the quasipotential approach, introducing a novel formal operation to relate the light-front bound and scattering states to the corresponding Bethe-Salpeter amplitudes. With this machinery at hand, we explore in Sec. III the light-front WTI and the conservation of the electromagnetic current. In Sec. IV, it is shown that truncation of the expansion in the quasipotential leads to a nonconserved current. In Sec. V, we fix this problem and the correction is found that restores current conservation at any given order in the quasipotential expansion. The corresponding WTI valid for that order is also given. In Sec. VI, the formalism is realized explicitly in one test case for the ladder Bethe-Salpeter equation. The current operator and the corresponding WTI are calculated in detail in the lowest nontrivial order of the quasipotential expansion. Our conclusions are summarized in Sec. VII. All detailed formal derivations are left to Appendices A, B, C, D, E, F, and G.

\section{QUASIPOTENTIAL APPROACH TO LIGHT-FRONT}

In the following we discuss the quasipotential expansion tailored to project on the light-front the field-theoretic Bethe-Salpeter equation (BSE) [2], which describes a relativistic two-particle system with bosonic constituents. First, we introduce our notation, the BS formalism, and the conserved four-dimensional electromagnetic current. Second, we review the quasipotential approach to perform the projection on the light-front.

\section{A. BS formalism, current, and Ward-Takahashi identity}

The transition matrix $(T)$ of two-particle scattering is described by an inhomogeneous BSE

$$
T=V+V G_{0} T,
$$

where $G_{0}$ is the disconnected Green's function for two 
particles and $V$ stands for the complete interaction, irreducible with respect to two-particle propagation. $G_{0}$ is reduced to the Green's function of two noninteracting particles,

$$
G_{0}=\frac{i}{\hat{k}_{1}^{2}-m_{1}^{2}+i o} \frac{i}{\hat{k}_{2}^{2}-m_{2}^{2}+i o},
$$

by neglecting self-energy parts. The off-mass-shell momentum operator acting on the coordinates of particle $i$ with mass $m_{i}$ is $\hat{k}_{i}^{\mu}$, the hat on the variable indicating the operator character. The BS amplitude of a scattering state of total 4-momentum $K$ is

$$
\left.\left.\left.\mid \Psi^{+}\right)=\mid \Psi_{0}\right)+G_{0} V \mid \Psi^{+}\right),
$$

where the round ket indicates the dependence on 4dimensional coordinates of all particles. $\left.\mid \Psi^{+}\right)$carries a 4dimensional $\delta$-function in momentum space,

$$
\left.\left\langle K^{\prime}\right| \Psi^{+}\right)=\delta\left(K^{\prime}-K\right)\left|\Psi^{+}\right\rangle .
$$

For a bound state with total 4-momentum $K_{B}, K_{B}^{2}=M_{B}^{2}$, the BS amplitude is solution of the homogeneous equation,

$$
\left.\left.\mid \Psi_{B}\right)=G_{0} V \mid \Psi_{B}\right),
$$

with the relation

$$
\left.\left\langle K^{\prime}\right| \Psi_{B}\right)=\delta\left(K^{\prime}-K_{B}\right)\left|\Psi_{B}\right\rangle .
$$

The normalization condition [3] has to be satisfied to fully determine $\left.\mid \Psi_{B}\right)$.

The total two-particle 4-momentum $K$ is conserved in Eqs. (1), (3), and (5). The operators $\mathcal{O}_{\alpha}=T, G_{0}$ or $V$, as well as the amplitude $\mid \Psi$ ) carry a 4-dimensional $\delta$-function in momentum space:

$$
\left\langle K^{\prime}\left|\mathcal{O}_{\alpha}\right| K\right\rangle=\delta\left(K^{\prime}-K\right) \mathcal{O}_{\alpha}(K) .
$$

After separating out the 4-dimensional delta function for total 4-momentum conservation, all reduced quantities, $\left|\Psi^{+}\right\rangle,\left|\Psi_{B}\right\rangle$, and $\mathcal{O}_{\alpha}(K)$, depend parametrically on $K$, even if not explicit. The reduced BS amplitude $|\Psi\rangle$ and 4-dimensional operator $\mathcal{O}_{\alpha}(K)$ are functions of the internal variables depicted as the individual 4-dimensional momentum $k^{\mu}$. For convenience we have used the bra-ket notation to represent functions of $k^{\mu}$.

We consider an electromagnetic current operator,

$$
\mathcal{J}^{\mu}(Q)=\mathcal{J}_{0}^{\mu}(Q)+\mathcal{J}_{I}^{\mu}(Q),
$$

which has a free term and another one that carries the interaction, with a 4-momentum transfer $Q=K_{f}-K_{i}$, defined as the difference between the final and initial total momentum of the two-body system. For example, it may contain crossed diagrams or charged boson exchanges.

The current is applied for elastic or inelastic processes. The initial state is described by a Bethe-Salpeter amplitude $\left|\Psi_{i}\right\rangle$ and the final one by $\left|\Psi_{f}\right\rangle$. The matrix element for the process $\left\langle\Psi_{f}\left|\mathcal{J}^{\mu}(Q)\right| \Psi_{i}\right\rangle$ can first be derived from the
Bethe-Salpeter amplitude $|\Psi\rangle$ of the bound or scattering states.

The current operator for the system of two charged particles 1 and 2 satisfies a Ward-Takahashi identity [3,14],

$$
Q_{\mu} \mathcal{J}^{\mu}(Q)=\left[G^{-1}, \hat{e}_{1}\right]+(1 \leftrightarrow 2),
$$

where $\hat{e}_{i}$ is the charge operator for particle $i$, and $G$ is the full Green's function of the interacting two-particle system, i.e., solution of

$$
G(K)=G_{0}(K)+G_{0}(K) V(K) G(K) .
$$

The charge operator has matrix elements

$$
\left\langle k_{i}\left|\hat{e}_{i}\right| p_{i}\right\rangle=e_{i} \delta^{4}\left(k_{i}-p_{i}-Q\right) .
$$

Current conservation is stated as

$$
\begin{aligned}
Q_{\mu}\left\langle\Psi_{f}\left|\mathcal{J}^{\mu}(Q)\right| \Psi_{i}\right\rangle= & \left\langle\Psi_{f}\left|G^{-1}\left(K_{f}\right) \hat{e}_{1}-\hat{e}_{1} G^{-1}\left(K_{i}\right)\right| \Psi_{i}\right\rangle \\
& +(1 \leftrightarrow 2),
\end{aligned}
$$

where we have explicitly shown the total 4-momentum arguments of the full Green's functions. Taking into account that the BS amplitude for bound and scattering states is a solution of

$$
G^{-1}\left(K_{\lambda}\right)\left|\Psi_{\lambda}\right\rangle=0,
$$

with appropriate boundary conditions ( $\lambda$ labels initial or final states) and since $\left\langle\Psi_{\lambda}\right| G^{-1}\left(K_{\lambda}\right)=0$ is valid as well, current conservation, $Q_{\mu}\left\langle\Psi_{f}\left|\mathcal{J}^{\mu}(Q)\right| \Psi_{i}\right\rangle=0$, follows.

\section{B. Projection on the light-front and QP approach}

We review, in the following, the main steps in using the quasipotential formalism as a tool to eliminate the relative light-front time between the particles. The well-known work of Ref. [5] shows how to calculate the transition matrix $T(K)$ and the Bethe-Salpeter amplitude $\left|\Psi_{B}\right\rangle$ of the covariant BSE from an auxiliary Green's function $\tilde{G}_{0}(K)$, which pave the path to perform the light-front projection. The auxiliary Green's function is given by [6]

$$
\tilde{G}_{0}(K):=G_{0}(K)\left|g_{0}^{-1}(K)\right| G_{0}(K),
$$

where $g_{0}(K)=\left|G_{0}(K)\right|$ is the global light-front time propagator of two-particles for $K^{+}>0$ and the vertical bar | indicates that the dependence on $k_{1}^{-}$is integrated out (see Appendix A). The bar on the left of the Green's function represents integration on $k_{1}^{-}$in the bra-state, the bar on the right in the ket-state. (The reader should be aware that the present notation cannot be confused with the absolute value.) Some properties are a direct consequence of Eq. (14):

$$
\begin{gathered}
\tilde{G}_{0}(K)\left|=G_{0}(K)\right|, \quad\left|\tilde{G}_{0}(K)=\right| G_{0}(K), \\
\left|\tilde{G}_{0}(K)\right|=\left|G_{0}(K)\right| .
\end{gathered}
$$

The transition matrix is the solution of the following equation with auxiliary Green's function 


$$
T(K)=W(K)+W(K) \tilde{G}_{0}(K) T(K),
$$

provided that the driving term satisfies

$$
W(K)=V(K)+V(K) \Delta_{0}(K) W(K) .
$$

The notation

$$
\begin{aligned}
\Delta_{0}(K) & =G_{0}(K)-G_{0}(K)\left|g_{0}(K)^{-1}\right| G_{0}(K) \\
& =G_{0}(K)-\tilde{G}_{0}(K)
\end{aligned}
$$

was used here.

The light-front transition matrix is defined through

$$
t(K):=g_{0}(K)^{-1}\left|G_{0}(K) T(K) G_{0}(K)\right| g_{0}(K)^{-1},
$$

which retains all the physics of $T(K)$. The relation between $T$ and $t$ is found by iterating the integral equation (16) once,

$$
\begin{aligned}
T(K)= & W(K)+W(K)\left[\tilde{G}_{0}(K)\right. \\
& \left.+\tilde{G}_{0}(K) T(K) \tilde{G}_{0}(K)\right] W(K) .
\end{aligned}
$$

Then, one substitutes $\tilde{G}_{0}(K)$ by Eq. (14) and the light-front scattering matrix by Eq. (19), getting

$$
\begin{aligned}
T(K)= & W(K)+W(K) G_{0}(K) \mid\left[g_{0}(K)^{-1}+t(K)\right] \\
& \times \mid G_{0}(K) W(K) .
\end{aligned}
$$

The light-front scattering matrix $t(K)$ is a solution of

$$
t(K)=w(K)+w(K) g_{0}(K) t(K),
$$

easily derived from Eq. (21), with the driving term $w(K)$ closely related to the 4-dimensional interaction $W(K)$ of Eq. (17), according to

$$
w(K):=g_{0}(K)^{-1}\left|G_{0}(K) W(K) G_{0}(K)\right| g_{0}(K)^{-1} .
$$

The series expansion in $W(K)$, and consequently in $w(K)$, amounts to consider higher Fock states in the virtual intermediate state propagations. The use of $\tilde{G}_{0}(K)$ and the corresponding integral equation for $W(K)$, Eq. (17), allows only for light-front two-body irreducible terms. The expansion in the Fock space is rapidly converging within a given covariant model as has been shown for bosonic models (see [6,21-24]). Furthermore, it was also found [25] that the probability of higher Fock-state components in the light-front wave function decreases fast in the WickCutkosky model. In fermionic models the light-front BS equation was studied with the lowest order kernel and problems due to the divergence on transverse momentum were quite subtle $[7,26,27]$. We postpone the discussion of fermionic systems for a future work.

The interacting light-front global propagator in the valence sector is $g(K):=|G(K)|$, which is derived from the full Green's function as

$$
|G(K)|=\left|G_{0}(K)\right|+\left|G_{0}(K) T(K) G_{0}(K)\right|,
$$

where the bar operation is taken on the left and right of the above equation. Using the definition of the LF scattering matrix, Eq. (19), one gets

$$
\begin{aligned}
g(K) & =g_{0}(K)+g_{0}(K) t(K) g_{0}(K) \\
& =g_{0}(K)+g_{0}(K) w(K) g(K) .
\end{aligned}
$$

The projection on the light-front eliminates the relative time between the particles allowing only global propagation in the $x^{+}$time.

The valence component, $|\phi\rangle$, of the light-front wave function for bound and scattering states is a solution of

$$
g(K)^{-1}|\phi\rangle=0,
$$

with an appropriate boundary condition. The above equation corresponds to the LF projection of the BS equation and related amplitudes. The correspondence between the valence wave function and the BS amplitude is given by

$$
\left.|\Psi\rangle=\left[1+\Delta_{0}(K) W(K)\right] G_{0}(K)\left|g_{0}(K)^{-1}\right| \phi\right\rangle,
$$

valid equally well for bound and scattering states, as shown in Appendix B.

The following identity for the reverse LF-time operation,

$$
G(K)\left|g(K)^{-1}=\left[1+\Delta_{0}(K) W(K)\right] G_{0}(K)\right| g_{0}(K)^{-1},
$$

demonstrated in Appendix C, valid for bound and scattering states, allows one to write Eq. (27) in a compact form,

$$
\left.|\Psi\rangle=G(K)\left|g(K)^{-1}\right| \phi\right\rangle,
$$

useful for formal manipulations. Conversely, the LF wave function is $|\phi\rangle=\| \Psi\rangle$.

The valence component of the light-front wave function for the bound-state with a four momentum $K_{B}$ is a solution of the homogenous equation,

$$
\left|\phi_{B}\right\rangle=g_{0}\left(K_{B}\right) w\left(K_{B}\right)\left|\phi_{B}\right\rangle,
$$

while the scattering state is the solution of the inhomogeneous equation,

$$
\left|\phi^{+}\right\rangle=\left|\phi_{0}\right\rangle+g_{0}(K) w(K)\left|\phi^{+}\right\rangle,
$$

with outgoing boundary condition. Although not indicated explicitly, the operators $g_{0}(K)$ and $w(K)$ have to be evaluated with $+\imath o$ prescription.

\section{LIGHT-FRONT WTI AND EM CURRENT CONSERVATION}

The three-dimensional light-front electromagnetic current operator is defined by identifying the matrix elements of the four-dimensional current between states described by BS amplitudes with the corresponding threedimensional ones. In a previous work [6], the current operator was only derived for bound states (see also [8]). However, the Bethe-Salpeter amplitude for bound and scattering states can be expressed by Eq. (29) (see 
Appendix C) which implies that the current operator found for bound states can be used with scattering states as well.

The matrix element of the electromagnetic current operator $\mathcal{J}^{\mu}(Q)$, describing an elastic or inelastic processes for interacting two-body systems, is expressed by $\left\langle\Psi_{f}\left|\mathcal{J}^{\mu}(Q)\right| \Psi_{i}\right\rangle$. The matrix element for the transition between the initial $(i)$ and final $(f)$ states can be obtained from the LF wave functions $\left|\phi_{i}\right\rangle$ and $\left|\phi_{f}\right\rangle$ by

$$
\left\langle\Psi_{f}\left|\mathcal{J}^{\mu}(Q)\right| \Psi_{i}\right\rangle=\left\langle\phi_{f}\left|j^{\mu}\left(K_{f}, K_{i}\right)\right| \phi_{i}\right\rangle,
$$

with the effective current operator in three-dimensional space given by

$$
j^{\mu}\left(K_{f}, K_{i}\right):=g\left(K_{f}\right)^{-1}\left|G\left(K_{f}\right) \mathcal{J}^{\mu}(Q) G\left(K_{i}\right)\right| g\left(K_{i}\right)^{-1} .
$$

The final state light-front wave function for scattering energies is a solution of $g\left(K_{f}\right)^{-1}\left|\phi_{f}\right\rangle=0$ with ingoing boundary condition.

Because of the identity (28), the effective current operator of Eq. (33) acting on the valence wave function, given by

$$
\begin{aligned}
j^{\mu}\left(K_{f}, K_{i}\right)= & g_{0}\left(K_{f}\right)^{-1} \mid G_{0}\left(K_{f}\right)\left[1+W\left(K_{f}\right) \Delta_{0}\left(K_{f}\right)\right] \\
& \times \mathcal{J}^{\mu}(Q)\left[1+\Delta_{0}\left(K_{i}\right) W\left(K_{i}\right)\right] \\
& \times G_{0}\left(K_{i}\right) \mid g_{0}\left(K_{i}\right)^{-1},
\end{aligned}
$$

matches the expression previously found valid for bound states [6]. The general form of Eq. (33), derived within the quasipotential formalism, is the same as the one found in Ref. [8].

The Ward-Takahashi identity of Eq. (9) is written as

$$
Q_{\mu} G\left(K_{f}\right) \mathcal{J}^{\mu}(Q) G\left(K_{i}\right)=\left[\hat{e}_{1}, G\right]+(1 \leftrightarrow 2),
$$

which permits the LF projection operation as

$$
Q_{\mu}\left|G\left(K_{f}\right) \mathcal{J}^{\mu}(Q) G\left(K_{i}\right)\right|=\left|\left[\hat{e}_{1}, G\right]\right|+(1 \leftrightarrow 2) .
$$

Using Eq. (D1), and multiplying each side by $g^{-1}$, one gets the LF Ward-Takahashi identity:

$$
Q^{\mu} j_{\mu}\left(K_{f}, K_{i}\right)=\left[g^{-1}, \hat{e}_{\mathrm{LF}}\right],
$$

where the LF charge operator has matrix elements given by

$$
\begin{aligned}
\left\langle k_{i}^{+}, \vec{k}_{i \perp}\left|\hat{e}_{i, \mathrm{LF}}\right| p_{i}^{+}, \vec{p}_{i \perp}\right\rangle= & e_{i} \delta\left(k_{i}^{+}-p_{i}^{+}-Q^{+}\right) \\
& \times \delta^{2}\left(\vec{k}_{i \perp}-\vec{p}_{i \perp}-\vec{Q}_{\perp}\right),
\end{aligned}
$$

as derived in Appendix D. [A proof of the light-front WTI (37) without using the reverse LF-time operation is given in Appendix E.]

The matrix elements of the current operator are conserved,

$$
Q_{\mu}\left\langle\phi_{f}\left|j^{\mu}\left(K_{f}, K_{i}\right)\right| \phi_{i}\right\rangle=0,
$$

as follows from Eq. (37) and from the eigenvalue equation satisfied by the LF states $\phi_{f}$ and $\phi_{i}$. This just confirms the consistence of the formalism. Equation (39) is a trivial consequence of the 4-dimensional current conservation as the matrix elements are identical and expressed by (32).

\section{TRUNCATION AND CURRENT NONCONSERVATION}

In the practical evaluation of the quasipotential (17) only few terms are kept hoping that they are sufficient for the physical problem at hand. The solution of $W$ by iteration of Eq. (17) gives

$$
\begin{aligned}
& W(K)=V(K) \sum_{i=0}^{\infty}\left[\Delta_{0}(K) V(K)\right]^{i} \\
& W(K)=W_{1}(K)+W_{2}(K)+\cdots,
\end{aligned}
$$

where $W_{n}=V(K)\left[\Delta_{0}(K) V(K)\right]^{n-1}$ with $n \geq 1$.

The truncation of the infinite series presented as

$$
W^{(n)}(K)=\sum_{i=1}^{n} W_{i}(K)
$$

and the corresponding light-front interaction,

$$
w^{(n)}(K)=\sum_{i=1}^{n} g_{0}(K)^{-1}\left|G_{0}(K) W_{i}(K) G_{0}(K)\right| g_{0}(K)^{-1},
$$

includes only a finite number of light-front Fock states in the intermediate state propagations. For example, in the ladder approximation for $n=2$, states up to 4-particles are taken into account in the kernel of the light-front BS equation (see [6]).

Truncated quantities as Green's function $G_{n}(K)$ and $g_{n}(K)$ are calculated with the truncated quasipotential. Then one is tempted to compute the truncated current operator directly from Eq. (33), retaining the truncated quasipotential $W^{(n)}$ in the calculation of the Green's function, yielding

$$
j_{\mu}^{(n)}\left(K_{f}, K_{i}\right)=g_{n}^{-1}\left(K_{f}\right)\left|G_{n}\left(K_{f}\right) \mathcal{J}_{\mu}(Q) G_{n}\left(K_{i}\right)\right| g_{n}^{-1}\left(K_{i}\right) .
$$

However this current is not conserved, as we are going to show in the following.

From Eq. (28), truncated at order $n$ it is also possible to write Eq. (43) as

$$
\begin{aligned}
j_{\mu}^{(n)}\left(K_{f}, K_{i}\right)= & g_{0}^{-1}\left(K_{f}\right) \mid G_{0}\left(K_{f}\right)\left[1+W^{(n)}\left(K_{f}\right) \Delta_{0}\left(K_{f}\right)\right] \\
& \times \mathcal{J}_{\mu}(Q)\left[1+\Delta_{0}\left(K_{i}\right) W^{(n)}\left(K_{i}\right)\right] \\
& \times G_{0}\left(K_{i}\right) \mid g_{0}^{-1}\left(K_{i}\right) .
\end{aligned}
$$

This current cannot be conserved, since 


$$
\begin{aligned}
Q^{\mu}\left\langle\phi_{f}^{(n)}\left|j_{\mu}^{(n)}\left(K_{f}, K_{i}\right)\right| \phi_{i}^{(n)}\right\rangle & =\left\langle\Psi_{f}^{(n)}\left|Q^{\mu} \mathcal{J}_{\mu}(Q)\right| \Psi_{i}^{(n)}\right\rangle \\
& =\left\langle\Psi_{f}^{(n)}\left|\left[\hat{e}, G^{-1}\right]\right| \Psi_{i}^{(n)}\right\rangle \neq 0,
\end{aligned}
$$

once $G^{-1}\left(K_{i}\right)\left|\Psi_{i}^{(n)}\right\rangle \neq 0$ and $\left\langle\Psi_{f}^{(n)}\right| G^{-1}\left(K_{f}\right) \neq 0$.

The deep structural reason for the nonconservation of the truncated current is: a WTI of the desired form cannot hold, i.e.,

$$
Q^{\mu} j_{\mu}^{(n)}\left(K_{f}, K_{i}\right) \neq\left[\hat{e}_{\mathrm{LF}}, g_{n}^{-1}\right],
$$

since $j_{\mu}^{(n)}$ contains terms of $\mathcal{O}\left[\left(w^{(n)}\right)^{2}\right]$, i.e., terms in $V$ up to order $2 n$, whereas $g_{n}^{-1}$ is of $\mathcal{O}\left[\left(w^{(n)}\right)\right]$, as it contains terms in $V$ up to order $n$.

\section{RESTORATION OF CURRENT CONSERVATION}

The identification of the reason for current nonconservation also suggests a strategy for restoration of current conservation. We remove from $j_{\mu}^{(n)}$ all terms of order higher than $n$ in $V$,

$$
j_{\mu}^{c(n)}:=j_{\mu}^{(n)}+\Delta j_{\mu}^{(n)},
$$

$\Delta j_{\mu}^{(n)}$ is just the correction which carries out that removal. The explicit forms of $j_{\mu}^{c(n)}$ and $\Delta j_{\mu}^{(n)}$ are given in Appendices $\mathrm{F}$ and $\mathrm{G}$, respectively. The proof that $j_{\mu}^{c(n)}$ indeed satisfies the desired WTI,

$$
Q^{\mu} j_{\mu}^{c(n)}\left(K_{f}, K_{i}\right)=\left[g_{n}^{-1}, \hat{e}_{\mathrm{LF}}\right],
$$

is performed in detail in Appendix F. We simply present the resulting expression here:

$$
\begin{aligned}
j^{c \mu(n)}= & g_{0}^{-1} \mid G_{0}\left[\mathcal{J}^{\mu}(Q)+\sum_{i=1}^{n-1}\left(W_{i} \Delta_{0} \mathcal{J}^{\mu}(Q)\right.\right. \\
& \left.+\mathcal{J}^{\mu}(Q) \Delta_{0} W_{i}\right)+\sum_{i=2}^{n-1} \sum_{j=1}^{i-1} W_{j} \Delta_{0} \mathcal{J}^{\mu}(Q) \Delta_{0} W_{i-j} \\
& +W_{n} \Delta_{0} \mathcal{J}_{0}^{\mu}(Q)+\mathcal{J}_{0}^{\mu}(Q) \Delta_{0} W_{n} \\
& \left.+\sum_{i=1}^{n-1} W_{i} \Delta_{0} \mathcal{J}_{0}^{\mu}(Q) \Delta_{0} W_{n-i}\right] G_{0} \mid g_{0}^{-1},
\end{aligned}
$$

where it is understood, but not explicitly written, that operators appearing at the left and at the right of the current depend on $K_{f}$ and $K_{i}$, respectively.

By comparing Eq. (49) with the equations presented in Appendix G, we note the limits

$$
\lim _{n \rightarrow \infty} \Delta j_{\mu}^{(n)}=0 \quad \lim _{n \rightarrow \infty} j_{\mu}^{c(n)}=j_{\mu}=\lim _{n \rightarrow \infty} j_{\mu}^{(n)},
$$

but now the conserved current $j_{\mu}^{c(n)}$ is found for each step of truncation.

Of course, the correction $\Delta j_{\mu}^{(n)}$ induces a fourdimensional correction term according to

$$
\left\langle\Psi_{f}^{(n)}\left|\Delta \mathcal{J}_{\mu}^{(n)}(Q)\right| \Psi_{i}^{(n)}\right\rangle:=\left\langle\phi_{f}^{(n)}\left|\Delta j_{\mu}^{(n)}\left(K_{f}, K_{i}\right)\right| \phi_{i}^{(n)}\right\rangle,
$$

which leads to the definition of the matrix elements of a corresponding 4-dimensional conserved current:

$$
\left\langle\Psi_{f}^{(n)}\left|\mathcal{J}_{\mu}^{c(n)}(Q)\right| \Psi_{i}^{(n)}\right\rangle:=\left\langle\Psi_{f}^{(n)}\left|\mathcal{J}_{\mu}(Q)+\Delta \mathcal{J}_{\mu}^{(n)}(Q)\right| \Psi_{i}^{(n)}\right\rangle .
$$

That matrix element of the 4-dimensional current, $\left\langle\Psi_{f}^{(n)}\left|\mathcal{J}_{\mu}^{c(n)}(Q)\right| \Psi_{i}^{(n)}\right\rangle$, can be related to the corresponding light-front one, i.e.,

$$
\left\langle\Psi_{f}^{(n)}\left|\mathcal{J}_{\mu}^{c(n)}(Q)\right| \Psi_{i}^{(n)}\right\rangle:=\left\langle\phi_{f}^{(n)}\left|j_{\mu}^{c(n)}\left(K_{f}, K_{i}\right)\right| \phi_{i}^{(n)}\right\rangle,
$$

and it is therefore also conserved at each step of truncation, i.e.,

$$
Q^{\mu}\left\langle\Psi_{f}^{(n)}\left|\mathcal{J}_{\mu}^{c(n)}(Q)\right| \Psi_{i}^{(n)}\right\rangle=0 .
$$

We have not found an explicit 4-dimensional form for $\Delta \mathcal{J}_{\mu}^{(n)}$, however, that form is conceptually unnecessary, since only matrix elements are required for the connection with observables.

To close this section, we stress that the implicit quasipotential expansion of the 4-dimensional EM current operator, Eq. (53), produces a conserved current at each order. This point emphasizes, in particular, the power of the formal construction of the quasipotential approach to perform the three-dimensional reduction of four-dimensional operators and amplitudes to the light-front hyperplane, without losing dynamical content. The quasipotential approach used in the present context furnishes a systematic tool to study consistently the dynamics and currents in light-front dynamics, connecting them to the 4dimensional counterparts as well.

The following section is devoted to illustrate in detail, in the example of the ladder Bethe-Salpeter equation, how the formal procedure derived in the context of the quasipotential approach manifests in a practical application. This give us the opportunity to comprehend how the theoretical tools developed so far can be explicitly realized.

\section{EXAMPLE: THE LADDER BETHE-SALPETER EQUATION}

In this section, we show one example that illustrates our procedure to construct the conserved current operator at order $n=1$ in the quasipotential expansion of $j^{c \mu(n)}$ in an actual case. Following our formalism, we derive explicitly the current operator of Eq. (49), in the lowest order of the quasipotential expansion, for the ladder Bethe-Salpeter equation describing a two-boson system.

The convergence of the quasipotential expansion, within the light-front framework, applied to solve the bosonic ladder Bethe-Salpeter equation is fast. This was shown numerically in Ref. [6], where they found that the relative difference between the bound-state masses and valence 
wave functions calculated with the quasipotential expansion in order $n=1$ and $n=2$ is, in general, small.

The Ward-Takahashi identity, given by Eq. (48), sums over the Lorentz index and therefore all components of the truncated electromagnetic current will be evaluated to verify current conservation. Here we calculate each Lorentz component of the truncated current operator, performing the projection of the corresponding fourdimensional quantity on the light-front, by integrating analytically on the minus momentum.

In this example we use the scalar Yukawa model, with the interaction Lagrangian given by

$$
\mathcal{L}_{I}=g_{S} \phi_{1}^{\dagger} \phi_{1} \sigma+g_{S} \phi_{2}^{\dagger} \phi_{2} \sigma
$$

where the bosons with fields $\phi_{1}$ and $\phi_{2}$ have masses $m_{1}$ and $m_{2}$, and charges $e_{1}$ and $e_{2}$. The chargeless exchanged boson with field $\sigma$ has mass $\mu$. The coupling constant is $g_{S}$.

The 4-dimensional current operator appropriate for the covariant ladder approximation is $\mathcal{J}^{\mu}(Q) \equiv \mathcal{J}_{0}^{\mu}(Q)$, with the matrix element of the free-current operator written as

$$
\begin{aligned}
\left\langle k_{1}\left|\mathcal{J}_{0}^{\mu}(Q)\right| p_{1}\right\rangle= & -2 \pi\left[e_{1}\left(k_{1}+p_{1}\right)^{\mu} \delta^{4}\left(k_{1}-p_{1}-Q\right)\right. \\
& \left.\times\left(\left(K_{f}-k_{1}\right)^{2}-m_{2}^{2}\right)\right] \\
& +\left[1 \rightarrow 2, k_{1} \rightarrow K_{f}-k_{1}, p_{1} \rightarrow K_{i}-p_{1}\right],
\end{aligned}
$$

where $Q^{\mu}=K_{f}^{\mu}-K_{i}^{\mu}$. The factor $(-2 \pi)$ is introduced in the current operator to make it compatible with Eq. (A4) for the 4-dimensional free Green's function, while the free WTI of Eq. (F2) is preserved.

The lowest order current operator for the interacting two-boson system in the ladder approximation is given by Eq. (49) with $n=1$,

$$
\begin{aligned}
j^{c \mu(1)}= & j^{c \mu(0)}+g_{0}^{-1} \mid G_{0}\left[W_{1} \Delta_{0} \mathcal{J}_{0}^{\mu}(Q)\right. \\
& \left.+\mathcal{J}_{0}^{\mu}(Q) \Delta_{0} W_{1}\right] G_{0} \mid g_{0}^{-1},
\end{aligned}
$$

where $W_{1}=V$ and

$$
j^{c \mu(0)}=g_{0}^{-1}\left|G_{0} \mathcal{J}_{0}^{\mu}(Q) G_{0}\right| g_{0}^{-1}
$$

is the free light-front current operator corresponding to $n=0$.

\section{A. Current operator in zeroth order}

We evaluate below the matrix elements of the interaction free light-front operator, $\left|G_{0} \mathcal{J}_{0}^{\mu}(Q) G_{0}\right|$. The matrix element of the free-current operator is derived from

$$
\begin{aligned}
\left\langle k_{1}^{\prime+} \vec{k}_{1 \perp}^{\prime}\left\|G_{0}\left(K_{f}\right) \mathcal{J}_{0}^{\mu}(Q) G_{0}\left(K_{i}\right)\right\| k_{1}^{+} \vec{k}_{1 \perp}\right\rangle= & -\frac{1}{(2 \pi)} \int d k_{1}^{-} \frac{e_{1}\left(2 k_{1}^{\mu}+Q^{\mu}\right) \delta\left(k_{1}^{\prime+}-k_{1}^{+}-Q^{+}\right) \delta^{2}\left(\vec{k}_{1 \perp}^{\prime}-\vec{k}_{1 \perp}-\vec{Q}_{\perp}\right)}{k_{1}^{\prime+} k_{1}^{+}\left(k_{1}^{-}+Q^{-}-\frac{\vec{k}_{1 \perp}^{\prime 2}+m_{1}^{2}-i o}{k_{1}^{\prime+}}\right)\left(k_{1}^{-}-\frac{\vec{k}_{1 \perp}^{2}+m_{1}^{2}-i o}{k_{1}^{+}}\right)} \\
& \times \frac{1}{\left(K_{i}^{+}-k_{1}^{+}\right)\left(K_{i}^{-}-k_{1}^{-}-\frac{\left(\vec{K}_{i \perp}-\vec{k}_{1 \perp}\right)^{2}+m_{2}^{2}-i o}{K_{i}^{+}-k_{1}^{+}}\right)}+1 \leftrightarrow 2 .
\end{aligned}
$$

Integrating over $k_{1}^{-}$and assuming that $K_{i}^{+}>0$ and $Q^{+} \geq 0$, without loss of generality, one gets that

$$
\begin{aligned}
& \left\langle k_{1}^{\prime+} \vec{k}_{1 \perp}^{\prime}\left\|G_{0}\left(K_{f}\right) \mathcal{J}_{0}^{\mu}(Q) G_{0}\left(K_{i}\right)\right\| k_{1}^{+} \vec{k}_{1 \perp}\right\rangle \\
& =i \theta\left(k_{1}^{+}\right) \theta\left(k_{1}^{\prime+}\right) \theta\left(K_{i}^{+}-k_{1}^{+}\right) \theta\left(K_{f}^{+}-k_{1}^{\prime+}\right) \\
& \quad \times \frac{e_{1}\left(K_{f}+K_{i}-\left(K_{f}-k_{1}^{\prime}\right)_{\text {on }}-\left(K_{i}-k_{1}\right)_{\text {on }}\right)^{\mu} \delta\left(k_{1}^{\prime+}-k_{1}^{+}-Q^{+}\right) \delta^{2}\left(\vec{k}_{1 \perp}^{\prime}-\vec{k}_{1 \perp}-\vec{Q}_{\perp}\right)}{k_{1}^{\prime+}\left(K_{i}^{+}-k_{1}^{+}\right) k_{1}^{+}\left(K_{f}^{-}-k_{\text {lon }}^{\prime-}-\left(K_{f}-k_{1}^{\prime}\right)_{\text {on }}^{-}+i o\right)\left(K_{i}^{-}-k_{1 \mathrm{on}}^{-}-\left(K_{i}-k_{1}\right)_{\mathrm{on}}^{-}+i o\right)}+1 \leftrightarrow 2 .
\end{aligned}
$$

The on-minus-shell values of the individual momenta are

$$
\begin{aligned}
& k_{1 \text { on }}^{\prime-}=\frac{\vec{k}_{1 \perp}^{2}+m_{1}^{2}}{k_{1}^{\prime+}} \\
& k_{1 \text { on }}^{-}=\frac{\vec{k}_{1 \perp}^{2}+m_{1}^{2}}{k_{1}^{+}}
\end{aligned}
$$

$$
\begin{aligned}
& \left(K_{f}-k_{1}^{\prime}\right)_{\mathrm{on}}^{-}=\frac{\left(\vec{K}_{f \perp}-\vec{k}_{1 \perp}^{\prime}\right)^{2}+m_{2}^{2}}{K_{f}^{+}-k_{1}^{+}} \\
& \left(K_{i}-k_{1}\right)_{\mathrm{on}}^{-}=\frac{\left(\vec{K}_{i \perp}-\vec{k}_{1 \perp}\right)^{2}+m_{2}^{2}}{K_{i}^{+}-k_{1}^{+}} .
\end{aligned}
$$

The identity $\left(K_{f}-k_{1}^{\prime}\right)_{\text {on }}^{-}=\left(K_{i}-k_{1}\right)_{\text {on }}^{-}$follows from three-dimensional momentum conservation. 
Taking into account the definition of $g_{0}(K)$ from Eq. (A7) and the light-front charge operator, Eq. (D3), the matrix element of the free-current operator is written as

$$
\begin{aligned}
\left\langle k_{1}^{\prime+} \vec{k}_{1 \perp}^{\prime}\left|j^{c \mu(0)}\right| k_{1}^{+} \vec{k}_{1 \perp}\right\rangle= & -i\left(\Delta_{f}^{(0)}+\Delta_{i}^{(0)}+k_{1 \text { on }}^{\prime}+k_{1 \text { on }}\right)^{\mu} \\
& \times\left(K_{i}^{+}-k_{1}^{+}\right) \\
& \times\left\langle k_{1}^{\prime+}, \vec{k}_{1 \perp}^{\prime}\left|\hat{e}_{1, \mathrm{LF}}\right| k_{1}^{+} \vec{k}_{1 \perp}\right\rangle \\
& +1 \leftrightarrow 2
\end{aligned}
$$

where we introduced $\Delta_{f}^{(0)}=K_{f}-k_{\text {lon }}^{\prime}-\left(K_{f}-k_{1}^{\prime}\right)_{\text {on }}$ and $\Delta_{i}^{(0)}=K_{i}-k_{1 \text { on }}-\left(K_{i}-k_{1}\right)_{\text {on }}$ for convenience.

The WTI for the free current is derived using that $Q=$ $\Delta_{f}^{(0)}-\Delta_{i}^{(0)}+k_{\text {lon }}^{\prime}-k_{\text {lon }}$, and noting that only the minus component of $\Delta_{f}$ and $\Delta_{i}$ are nonzero. Therefore, $Q$. $\left(\Delta_{f}^{(0)}+\Delta_{i}^{(0)}+k_{1 \text { on }}^{\prime}+k_{1 \text { on }}\right)$ is equal to $\Delta_{f}^{(0)-} k_{1}^{\prime+}-$ $\Delta_{i}^{(0)-} k_{1}^{+}$, which allows one immediately to write a 3dimensional form of the free WTI given by Eq. (F8).

\section{B. Current operator in first order}

The contribution of the interaction to the light-front current operator in lowest order comes from two-body irreducible amplitudes given by the second term in the right-hand side (rhs) of Eq. (57). Using Eq. (18) for $\Delta_{0}$ we write that:

$$
\begin{aligned}
j^{c \mu(1)}-j^{c \mu(0)}= & g_{0}^{-1}\left|G_{0} W^{(1)} G_{0} \mathcal{J}_{0}^{\mu}(Q) G_{0}\right| g_{0}^{-1} \\
& +g_{0}^{-1}\left|G_{0} \mathcal{J}_{0}^{\mu}(Q) G_{0} W^{(1)} G_{0}\right| g_{0}^{-1} \\
& -w^{(1)} g_{0} j^{c \mu(0)}-j^{c \mu(0)} g_{0} w^{(1)},
\end{aligned}
$$

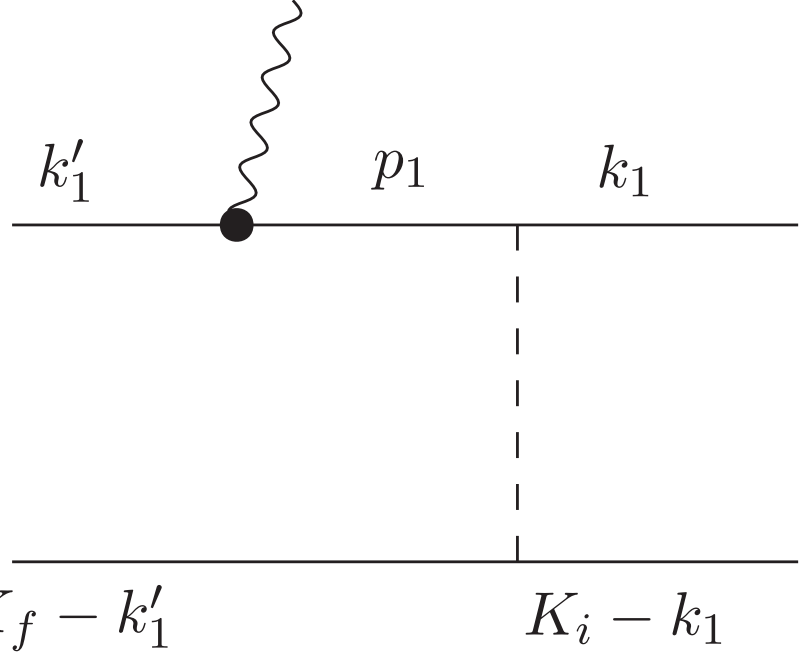

FIG. 1. Diagrammatic representation of the matrix elements of the 4-dimensional operator in Eq. (67).

where the effective interaction is given by $w^{(1)}=$ $g_{0}^{-1}\left|G_{0} W^{(1)} G_{0}\right| g_{0}^{-1}$ with $W^{(1)}=V$.

The two-body reducible parts of the first two terms in the rhs of Eq. (66), are canceled by the two last ones. The twobody reducible contributions are already included in the effective interaction used to obtain the valence wave function calculated from the first order quasipotential expansion of the ladder Bethe-Salpeter equation.

We choose to calculate in detail the second term in the rhs of Eq. (66), because it contains a pair production contribution for $Q^{+}>0$, while the other one does not have it, considering that choice of momentum transfer. Given that, we have to calculate the matrix elements

$$
\begin{aligned}
\left\langle k_{1}^{\prime+} \vec{k}_{1 \perp}^{\prime}\left\|G_{0} \mathcal{J}_{0}^{\mu}(Q) G_{0} V G_{0}\right\| k_{1}^{+} \vec{k}_{1 \perp}\right\rangle= & i e_{1}\left(\frac{i g_{S}}{2 \pi}\right)^{2} \int d k_{1}^{\prime-} d k_{1}^{-}\left(k_{1}^{\prime}+p_{1}\right)^{\mu} \frac{1}{k_{1}^{\prime+}\left(K_{f}^{+}-k_{1}^{\prime+}\right)} \frac{1}{\left(k_{1}^{\prime-}-\frac{\vec{k}_{1 \perp}^{\prime 2}+m_{1}^{2}-i o}{k_{1}^{\prime+}}\right)} \\
& \times \frac{1}{\left(K_{f}^{-}-k_{1}^{\prime-}-\frac{\left(\vec{K}_{f \perp}-\vec{k}_{1 \perp}^{\prime}\right)^{2}+m_{2}^{2}-i o}{K_{f}^{+}-k_{1}^{\prime+}}\right)} \frac{1}{\left(k_{1}^{+}-p_{1}^{+}\right) p_{1}^{+}} \\
& \times \frac{1}{\left(k_{1}^{-}-p_{1}^{-}-\frac{\left(\vec{k}_{1 \perp}-\vec{p}_{1 \perp}\right)^{2}+\mu^{2}-i o}{k_{1}^{++}-p_{1}^{+}}\right)} \frac{1}{\left(p_{1}^{-}-\frac{\vec{p}_{1 \perp}^{2}+m_{1}^{2}-i o}{p_{1}^{+}}\right)} \frac{1}{k_{1}^{+}\left(K_{i}^{+}-k_{1}^{+}\right)} \\
& \times \frac{1}{\left(k_{1}^{-}-\frac{\vec{k}_{1 \perp}^{2}+m_{1}^{2}-i o}{k_{1}^{+}}\right)} \frac{1}{\left(K_{i}^{-}-k_{1}^{-}-\frac{\left(\vec{K}_{i \perp}-\vec{k}_{1 \perp}\right)^{2}+m_{2}^{2}-i o}{K_{i}^{+}-k_{1}^{+}}\right)},
\end{aligned}
$$

represented by the Feynman diagram shown in Fig. 1. The momentum $p_{1}^{\mu}$ is $k_{1}^{\prime \mu}-Q^{\mu}$. In the same way, we have to compute the matrix elements of the first term in the rhs of Eq. (66). For the sake of simplicity, from now on we will just indicate the current for particle 1 .

The integrations over $k^{-}$in Eq. (67) are performed analytically using Cauchy's theorem with the conditions
$K_{i}^{+}>0$ and $Q^{+} \geq 0$. The integration is nonzero only for $K_{f}^{+}>k_{1}^{\prime+}>0$ and $K_{i}^{+}>k_{1}^{+}>0$. Altogether, four terms appear which are shown in Fig. 2. The two-body irreducible terms are represented by diagrams (a) and (b) of Fig. 1. The two-body reducible terms represented by diagrams (c) and (d) are canceled by the last term of Eq. (66). 


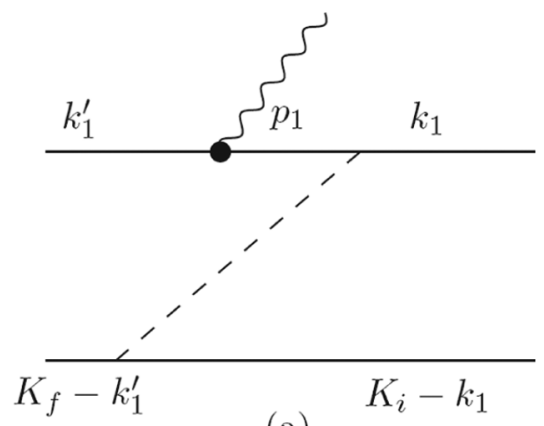

(a)

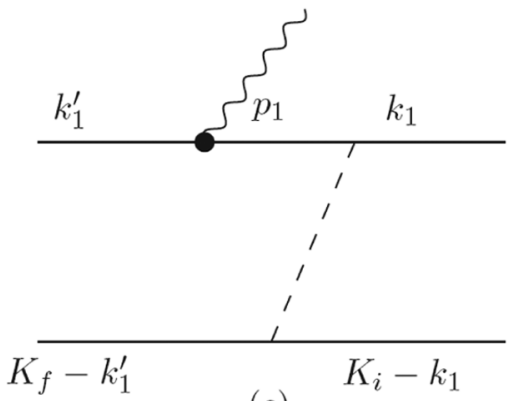

(c)

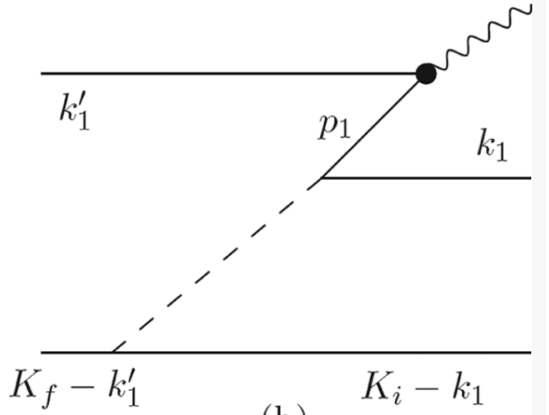

(b)

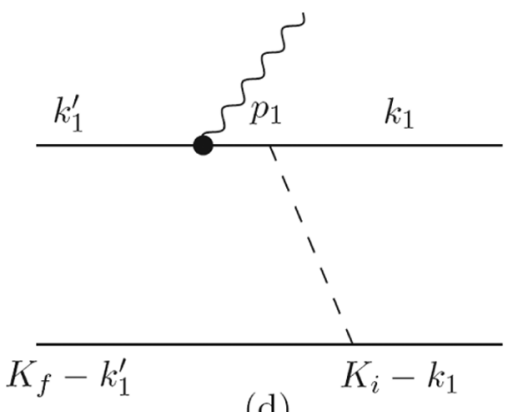

(d)

FIG. 2. Light-front time-ordered diagrams representing the matrix element of the two-body irreducible current operator obtained from the second term in the rhs of Eq. (66).

We are going to derive in detail the contributions to the current represented by diagrams (a) and (b). Diagram (a) gives a two-body current with intermediate three-body virtual state propagations before and after the photon absorption, in the kinematical range of $k_{1}^{\prime+}>$ $Q^{+} \geq 0$ and $k_{1}^{+}-k_{1}^{\prime+}+Q^{+}>0$. Diagram (b) represents the contribution to the current of the virtual photon decay in a boson-antiboson pair. The kinematical condition in this case is $0<k_{1}^{\prime+}<Q^{+}$. For the Drell-Yan-West frame, the expectation value of the current operator (b) vanishes due to the one-boson exchange and the corresponding soft endpoint behavior of the valence component. For a bound state it decreases quadratically with momentum fraction near the end points.

We perform analytically the integration of Eq. (67) in $k_{1}^{-}$ within the kinematical region $p_{1}^{+}=k_{1}^{\prime+}-Q^{+}>0$ and $k_{1}^{+}-k_{1}^{\prime+}+Q^{+}>0$ by evaluating the residue in the upper half of the $k_{1}^{-}$complex plane. The result is

$$
\begin{aligned}
\left\langle k_{1}^{\prime+} \vec{k}_{1 \perp}^{\prime}\left\|G_{0} \mathcal{J}_{0}^{\mu}(Q) G_{0} V G_{0}\right\| k_{1}^{+} \vec{k}_{1 \perp}\right\rangle_{(a)+(c)=} & e_{1} \frac{\left(i g_{S}\right)^{2}}{2 \pi} \theta\left(k_{1}^{+}\right) \theta\left(k_{1}^{\prime+}\right) \theta\left(K_{i}^{+}-k_{1}^{+}\right) \theta\left(K_{f}^{+}-k_{1}^{\prime+}\right) \theta\left(p_{1}^{+}\right) \theta\left(k_{1}^{+}-p_{1}^{+}\right) \\
& \times \theta\left(k_{1}^{\prime+}-Q^{+}\right) \int d k_{1}^{\prime-}\left(k_{1}^{\prime}+p_{1}\right)^{\mu} \frac{1}{k_{1}^{\prime+}\left(K_{f}^{+}-k_{1}^{\prime+}\right)} \frac{1}{\left(k_{1}^{\prime-}-\frac{\vec{k}_{1 \perp}^{\prime 2}+m_{1}^{2}-i o}{k_{1}^{\prime+}}\right)} \\
& \times \frac{1}{\left(K_{f}^{-}-k_{1}^{\prime-}-\frac{\left(\vec{K}_{f \perp}-\vec{k}_{1 \perp}^{\prime}\right)^{2}+m_{2}^{2}-i o}{K_{f}^{+}-k_{1}^{\prime+}}\right)} \frac{1}{\left(k_{1}^{+}-p_{1}^{+}\right) p_{1}^{+}} \\
& \times \frac{1}{\left(K_{f}^{-}-k_{1}^{\prime-}-\frac{\left(\vec{K}_{1 \perp}-\vec{k}_{1 \perp}\right)^{2}+m_{2}^{2}-i o}{K_{i}^{+}-k_{1}^{+}}-\frac{\left(\vec{k}_{1 \perp}-\vec{p}_{1 \perp}\right)^{2}+\mu^{2}-i o}{k_{1}^{+}-p_{1}^{+}}\right)} \\
& \times \frac{1}{\left(k_{1}^{\prime-}-Q^{-}-\frac{\vec{p}_{1 \perp}^{2}+m_{1}^{2}-i o}{p_{1}^{+}}\right)} \frac{1}{k_{1}^{+}\left(K_{i}^{+}-k_{1}^{+}\right)} \\
& \times \frac{1}{\left(K_{i}^{-}-\frac{\left(\vec{K}_{i \perp}-\vec{k}_{1 \perp}\right)^{2}+m_{2}^{2}-i o}{K_{i}^{+}-k_{1}^{+}}-\frac{\vec{k}_{1+}^{2}+m_{1}^{2}-i o}{k_{1}^{+}}\right)} .
\end{aligned}
$$


In order to separate the processes corresponding to diagrams (a) and (c) of Fig. 2, we make use of the identity

$$
\begin{aligned}
& \frac{1}{\left(K_{f}^{-}-k_{1}^{\prime-}-\frac{\left(\vec{K}_{i \perp}-\vec{k}_{1 \perp}\right)^{2}+m_{2}^{2}-i o}{K_{i}^{+}-k_{1}^{+}}-\frac{\left(\vec{k}_{1 \perp}-\vec{p}_{1 \perp}\right)^{2}+\mu^{2}-i o}{k_{1}^{+}-p_{1}^{+}}\right)} \frac{1}{\left(k_{1}^{\prime-}-Q^{-}-\frac{\vec{p}_{1 \perp}^{2}+m_{1}^{2}-i o}{p_{1}^{+}}\right)} \\
& =\left(\frac{1}{K_{f}^{-}-k^{\prime-}-\frac{\left(\vec{K}_{i \perp}-\vec{k}_{1 \perp}\right)^{2}+m_{2}^{2}-i o}{K_{i}^{+}-k_{1}^{+}}-\frac{\left(\vec{k}_{1 \perp}-\vec{p}_{1 \perp}\right)^{2}+\mu^{2}-i o}{k_{1}^{+}-p_{1}^{+}}}+\frac{1}{k_{1}^{\prime-}-Q^{-}-\frac{\vec{p}_{1 \perp}^{2}+m_{1}^{2}-i o}{p_{1}^{+}}}\right) \\
& \quad \times \frac{1}{K_{i}^{-}-\frac{\vec{p}_{1 \perp}^{2}+m_{1}^{2}-i o}{p_{1}^{+}}-\frac{\left(\vec{k}_{1 \perp}-\vec{p}_{1 \perp}\right)^{2}+\mu^{2}-i o}{k_{1}^{+}-p_{1}^{+}}-\frac{\left(\vec{K}_{i \perp}-\vec{k}_{1 \perp}\right)^{2}+m_{2}^{2}-i o}{K_{i}^{+}-k_{1}^{+}}},
\end{aligned}
$$

and integrate Eq. (68) analytically. The first and second terms between the parentheses give, after $k^{\prime-}$ integration, diagrams (a) and (c), respectively. The contribution of diagram (c) to the current is two-body reducible and it is canceled out in Eq. (66), remaining only (a). The result is

$$
\left\langle k_{1}^{\prime+} \vec{k}_{1 \perp}^{\prime}\left|j_{\mu}^{c(1)}\right| k_{1}^{+} \vec{k}_{1 \perp}\right\rangle_{(a)}=i e_{1}\left(2 k_{1 \text { on }}^{\prime}-Q\right)_{\mu}\left(i g_{S}\right)^{2} \frac{\theta\left(k_{1}^{+}-p_{1}^{+}\right)}{\left(k_{1}^{+}-p_{1}^{+}\right) p_{1}^{+}} \frac{\theta\left(k_{1}^{\prime+}-Q^{+}\right)}{\Delta_{f}^{(1 a)-} \Delta_{i}^{(1 a)-}},
$$

where the combinations $\Delta_{f}^{(1 a)}$ and $\Delta_{i}^{(1 a)}$ of four momenta are

$$
\Delta_{f}^{(1 a)}=K_{f}-k_{1 \text { on }}^{\prime}-\left(K_{i}-k_{1}\right)_{\text {on }}-\left(k_{1}-p_{1}\right)_{\text {on }}+i o \quad \Delta_{i}^{(1 a)}=K_{i}-p_{1 \mathrm{on}}-\left(K_{i}-k_{1}\right)_{\mathrm{on}}-\left(k_{1}-p_{1}\right)_{\mathrm{on}}+i o,
$$

with the on-minus-shell definition of the respective momenta given by

$$
\begin{aligned}
k_{1 \text { on }}^{\prime-} & =\frac{\vec{k}_{1 \perp}^{\prime 2}+m_{1}^{2}}{k_{1}^{\prime+}} \\
\left(K_{i}-k_{1}\right)_{\text {on }}^{-} & =\frac{\left(\vec{K}_{i \perp}-\vec{k}_{1 \perp}\right)^{2}+m_{2}^{2}}{K_{i}^{+}-k_{1}^{+}} \\
\left(k_{1}-p_{1}\right)_{\text {on }}^{-} & =\frac{\left(\vec{k}_{1 \perp}-\vec{p}_{1 \perp}\right)^{2}+\mu^{2}}{k_{1}^{+}-p_{1}^{+}} \\
p_{1 \text { on }}^{-} & =\frac{\vec{p}_{1 \perp}^{2}+m_{1}^{2}}{p_{1}^{+}} .
\end{aligned}
$$

To complete the computation of the matrix element of the current in the kinematical region of $k_{1}^{\prime+}>Q^{+}$, we have to evaluate the two-body irreducible contribution from the first term in the rhs of Eq. (66), which is represented by diagram (e) given in Fig. 3. Following the same steps as before, one gets

$$
\begin{aligned}
\left\langle k_{1}^{\prime+} \vec{k}_{1 \perp}^{\prime}\left|j_{\mu}^{c(1)}\right| k_{1}^{+} \vec{k}_{1 \perp}\right\rangle_{(e)}= & i e_{1}\left(2 k_{1 \text { on }}+Q\right)_{\mu}\left(i g_{S}\right)^{2} \\
& \times \frac{\theta\left(k_{1}^{\prime+}-p_{1}^{+}\right)}{\left(k_{1}^{\prime+}-p_{1}^{+}\right) p_{1}^{+}} \\
& \times \frac{\theta\left(k_{1}^{\prime+}-Q^{+}\right)}{\Delta_{f}^{(1 e)-} \Delta_{i}^{(1 e)-}}
\end{aligned}
$$

where the combinations $\Delta_{f}^{(1 e)}$ and $\Delta_{i}^{(1 e)}$ of four momenta are

$$
\begin{aligned}
& \Delta_{f}^{(1 e)}=K_{f}-p_{1 \text { on }}-\left(K_{f}-k_{1}^{\prime}\right)_{\mathrm{on}}-\left(k_{1}^{\prime}-p_{1}\right)_{\mathrm{on}}+i o \\
& \Delta_{i}^{(1 e)}=K_{i}-k_{1 \mathrm{on}}-\left(K_{f}-k_{1}^{\prime}\right)_{\mathrm{on}}-\left(k_{1}^{\prime}-p_{1}\right)_{\mathrm{on}}+i o .
\end{aligned}
$$

The on-minus-shell definition of the respective momenta are given by

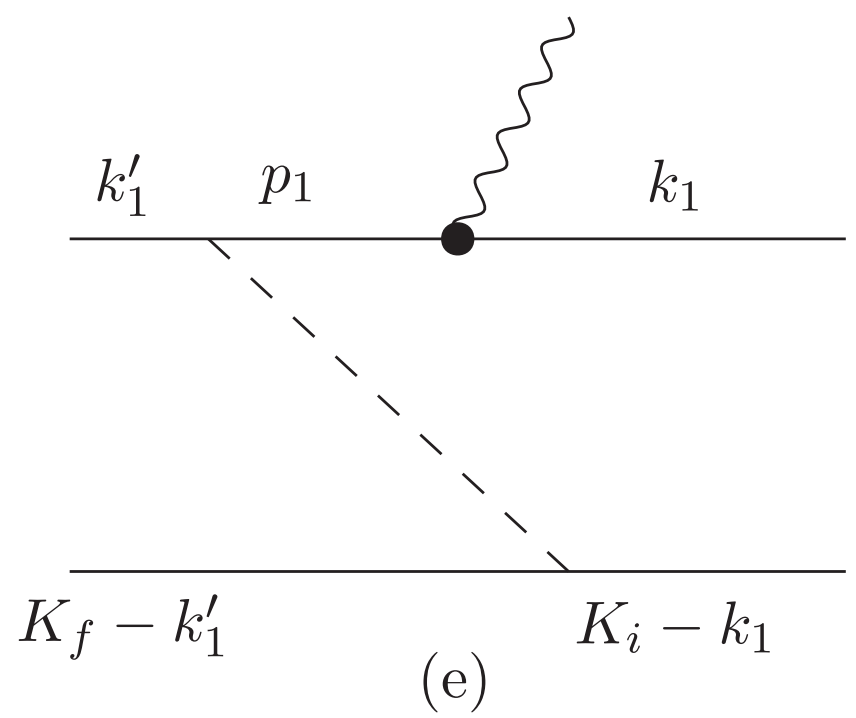

FIG. 3. Light-front time-ordered diagram representing the matrix element of the lowest order two-body irreducible current operator obtained from the first term in the rhs of Eq. (66). 


$$
\begin{aligned}
k_{1 \text { on }}^{-} & =\frac{\vec{k}_{1 \perp}^{2}+m_{1}^{2}}{k_{1}^{+}} \\
\left(K_{f}-k_{1}^{\prime}\right)_{\text {on }}^{-} & =\frac{\left(\vec{K}_{f \perp}-\vec{k}_{1 \perp}^{\prime}\right)^{2}+m_{1}^{2}}{K_{f}^{+}-k_{1}^{\prime+}} \\
\left(k_{1}^{\prime}-p_{1}\right)_{\text {on }}^{-} & =\frac{\left(\vec{k}_{1 \perp}^{\prime}-\vec{p}_{1 \perp}\right)^{2}+\mu^{2}}{k_{1}^{\prime+}-p_{1}^{+}} .
\end{aligned}
$$

We have introduced $\Delta_{f}^{(1 a)}, \Delta_{i}^{(1 a)}, \Delta_{f}^{(1 e)}$, and $\Delta_{i}^{(1 e)}$ to simplify the explicit proof of the validity of the WTI for the interacting current in lowest order. First we note that $\Delta_{i}^{(1 a)}-\Delta_{f}^{(1 a)}+k_{1 \text { on }}^{\prime}+p_{1 \text { on }}=2 k_{1 \text { on }}^{\prime}-Q$ and that $Q=$ $\Delta_{f}^{(1 a)}-\Delta_{i}^{(1 a)}+k_{1 \text { on }}^{\prime}-p_{1 \text { on }}$. Therefore,

$$
Q \cdot\left(2 k_{1 \text { on }}^{\prime}-Q\right)=\left(\Delta_{f}^{(1 a)-}-\Delta_{i}^{(1 a)-}\right) p_{1}^{+},
$$

and analogously one gets

$$
Q \cdot\left(2 k_{1 \text { on }}+Q\right)=\left(\Delta_{f}^{(1 e)-}-\Delta_{i}^{(1 e)-}\right) p_{1}^{+},
$$

which allows one to calculate the four-divergence of the current,

$$
\begin{aligned}
& Q \cdot\left\langle k_{1}^{\prime+} \vec{k}_{1 \perp}^{\prime}\left|j^{c(1)}\right| k_{1}^{+} \vec{k}_{1 \perp}\right\rangle_{(a)+(e)} \\
&=e_{1}\left[\left(\left(i g_{S}\right)^{2} \frac{\theta\left(k_{1}^{+}-p_{1}^{+}\right)}{\left(k_{1}^{+}-p_{1}^{+}\right)} \frac{i}{\Delta_{i}^{(1 a)-}}\right.\right. \\
&\left.+\left(i g_{S}\right)^{2} \frac{\theta\left(k_{1}^{\prime+}-p_{1}^{+}\right)}{\left(k_{1}^{\prime+}-p_{1}^{+}\right)} \frac{i}{\Delta_{i}^{(1 e)-}}\right) \\
&-\left(\left(i g_{S}\right)^{2} \frac{\theta\left(k_{1}^{+}-p_{1}^{+}\right)}{\left(k_{1}^{+}-p_{1}^{+}\right)} \frac{i}{\Delta_{f}^{(1 a)-}}\right. \\
&\left.\left.+\left(i g_{S}\right)^{2} \frac{\theta\left(k_{1}^{\prime+}-p_{1}^{+}\right)}{\left(k_{1}^{\prime+}-p_{1}^{+}\right)} \frac{i}{\Delta_{f}^{(1 e)-}}\right)\right] \theta\left(k_{1}^{++}-Q^{+}\right) .
\end{aligned}
$$

The first term within the square brackets is the interaction calculated at lowest order for total momentum $K_{i}$ and represented in Fig. 4. The second term is the corresponding interaction for total momentum $K_{f}$.

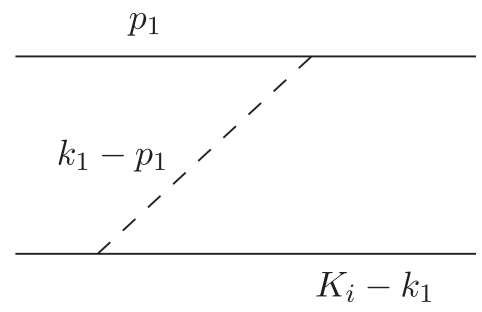

The commutator,

$$
\begin{aligned}
& \left\langle k_{1}^{\prime+} \vec{k}_{1 \perp}^{\prime}\left|Q \cdot j^{c(1)}\right| k_{1}^{+} \vec{k}_{1 \perp}\right\rangle_{(a)+(e)} \\
& \quad=\left\langle k_{1}^{\prime+} \vec{k}_{1 \perp}^{\prime}\left|\left[\hat{e}_{\mathrm{LF}}, w^{(1)}\right]\right| k_{1}^{+} \vec{k}_{1 \perp}\right\rangle \theta\left(k_{1}^{\prime+}-Q^{+}\right),
\end{aligned}
$$

expresses Eq. (78) at the level of operators for $K_{f}^{+}>k_{1}^{\prime+}>$ $Q^{+}$. The above expression was formally derived in Appendix F, however without any restriction. The missing piece that allows one to drop the constraint is the pair contribution to the current, expressed by diagram (b) of Fig. 2.

\section{Pair current operator}

The pair produced by the virtual photon decay for $Q^{+}>$ 0 , represented by diagram (b) in Fig. 2, contributes to the current operator in the kinematical region of $0<k_{1}^{\prime+}<$ $Q^{+}$. The matrix element of the current operator is derived from Eq. (68) evaluating the residue of the integrand in the lower half of the complex plane $k_{1}^{\prime-}$. In this situation only one pole appears at the position $k^{\prime-}=k_{\text {on }}^{\prime-}$. [Note that in Eq. (68) $\theta\left(k_{1}^{\prime+}-Q^{+}\right)$should be now substituted by $\theta\left(Q^{+}-k_{1}^{\prime+}\right)$.] The resulting matrix element of the current operator is

$$
\begin{aligned}
\left\langle k_{1}^{\prime+} \vec{k}_{1 \perp}^{\prime}\left|j_{\mu}^{c(1)}\right| k_{1}^{+} \vec{k}_{1 \perp}\right\rangle_{(b)}= & i e_{1}\left(2 k_{1 \text { on }}^{\prime}-Q\right)_{\mu}\left(i g_{S}\right)^{2} \\
& \times \frac{\theta\left(k_{1}^{+}-p_{1}^{+}\right)}{\left(k_{1}^{+}-p_{1}^{+}\right) p_{1}^{+}} \frac{\theta\left(Q^{+}-k_{1}^{\prime+}\right)}{\Delta_{f}^{(1 b)-} \Delta_{\gamma}^{(1 b)-}},
\end{aligned}
$$

where the combinations $\Delta_{f}^{(1 b)}$ and $\Delta_{\gamma}^{(1 b)}$ of four momenta are

$\Delta_{f}^{(1 b)}=K_{f}-k_{1 \text { on }}^{\prime}-\left(K_{i}-k_{1}\right)_{\text {on }}-\left(k_{1}-p_{1}\right)_{\text {on }}+i o$

$\Delta_{\gamma}^{(1 b)}=Q-k_{1 \text { on }}^{\prime}-\left(Q-k_{1}^{\prime}\right)_{\text {on }}+i o$,

with the on-minus-shell definition of the respective momenta given by the corresponding expressions in Eqs. (72) and (75). We have again introduced the four-vector quantities $\Delta_{f}^{(1 b)}$ and $\Delta_{\gamma}^{(1 b)}$ to simplify the identification of commutator in the WTI. To accomplish this we note that $2 k_{1 \text { on }}^{\prime}-Q=-\Delta_{\gamma}^{(1 b)}+k_{1 \text { on }}^{\prime}-\left(Q-k_{1}^{\prime}\right)_{\text {on }}$ and that $Q=$ $\Delta_{\gamma}^{(1 b)}+k_{1 \text { on }}^{\prime}+\left(Q-k_{1}^{\prime}\right)_{\text {on }}$. Therefore,

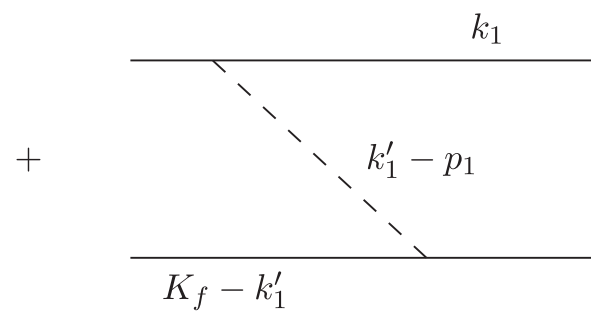

FIG. 4. Light-front time-ordered diagrams representing the matrix element of the lowest order interaction operator for a two-boson state with total momentum $K_{i}$. 


$$
Q \cdot\left(2 k_{1 \text { on }}^{\prime}-Q\right)=-\Delta_{\gamma}^{(1 b)-}\left(Q^{+}-k_{1}^{\prime+}\right),
$$

which allows one to calculate the four-divergence of the current operator as

$$
\begin{aligned}
Q \cdot\left\langle k_{1}^{\prime+} \vec{k}_{1 \perp}^{\prime}\left|j^{c(1)}\right| k_{1}^{+} \vec{k}_{1 \perp}\right\rangle_{(b)}= & -e_{1}\left(i g_{S}\right)^{2} \frac{\theta\left(k_{1}^{+}-p_{1}^{+}\right)}{\left(k_{1}^{+}-p_{1}^{+}\right)} \\
& \times \frac{i}{\Delta_{f}^{(1 b)-}} \theta\left(Q^{+}-k_{1}^{\prime+}\right) .
\end{aligned}
$$

This single term corresponds to the interaction calculated at lowest order for total momentum $K_{f}$ in the allowed kinematical region, where $p_{1}^{+}<0$. It is not possible to construct the effective interaction for total momentum $\mathrm{K}_{i}^{+}$represented in Fig. 4 because the kinematical constraints in the plus momentum forbids it. Therefore, Eq. (83) represents as well the commutator

$$
\begin{aligned}
& \left\langle k_{1}^{\prime+} \vec{k}_{1 \perp}^{\prime}\left|Q \cdot j^{c(1)}\right| k_{1}^{+} \vec{k}_{1 \perp}\right\rangle_{(b)} \\
& \quad=\left\langle k_{1}^{\prime+} \vec{k}_{1 \perp}^{\prime}\left|\left[\hat{e}_{\mathrm{LF}}, w^{(1)}\right]\right| k_{1}^{+} \vec{k}_{1 \perp}\right\rangle \theta\left(Q^{+}-k_{1}^{\prime+}\right) .
\end{aligned}
$$

Adding the above commutator with Eq. (79), the final formal result shown in Appendix $F$ is found. This simple example illustrates the power of the formal manipulations to construct the correct approximation of a conserved lightfront operator consistent with the effective interaction at each given order.
Another important aspect of this example is the necessity to consider all Lorentz components of the current operator, beyond the "good" one. The field theoretical model allows one to construct all four components of the current operator. Yet, the truncation in the quasipotential expansion, and correspondingly in Fock-space expansion, violates full covariance. This means that the matrix elements of the truncated electromagnetic current operator will have four-vector transformation properties only under kinematical boosts.

\section{Bad current and end-point singularity}

The minus component of the electromagnetic current of a scalar boson composed by scalar constituents in models with a pointlike vertices involves an end-point singularity that for $Q^{+} \rightarrow 0$ gives a nonvanishing contribution to the form factor [12]. The singular contribution to the minus component comes from the decay of the virtual photon in a boson-antiboson pair. In the present example, this raises the issue of the presence or not of an end-point singularity in the minus component of the pair current operator, Eq. (80), which could survive in the Drell-Yan-West frame.

Because of the kinematical constraint of $0<k_{1}^{\prime+}<Q^{+}$, terms like $1 / k_{1}^{\prime+}$ and $1 /\left(Q^{+}-k_{1}^{\prime+}\right)$ are singular and dominate over finite quantities, therefore for $Q^{+} \approx 0$ the pair current operator can be written as

$$
\begin{aligned}
\left\langle k_{1}^{\prime+} \vec{k}_{1 \perp}^{\prime}\left|j^{c(1)-}\right| k_{1}^{+} \vec{k}_{1 \perp}\right\rangle_{(b)} \approx & -2 i e_{1} \frac{\theta\left(Q^{+}-k_{1}^{\prime+}\right)\left(\vec{k}_{1 \perp}^{\prime 2}+m_{1}^{2}\right)}{k_{1}^{\prime+}\left(Q^{+}-k_{1}^{\prime+}\right)\left(Q^{-}-k_{1 \text { on }}^{\prime-}-\left(Q-k_{1}^{\prime}\right)_{\text {on }}^{-}+i o\right)}\left(i g_{S}\right)^{2} \\
& \times \frac{\theta\left(k_{1}^{+}\right)}{\left(k_{1}^{+}-k_{1}^{\prime+}+Q^{+}\right)\left(K_{f}^{-}-k_{1 \text { on }}^{\prime-}-\left(K_{i}-k_{1}\right)_{\text {on }}^{-}-\left(k_{1}-k_{1}^{\prime}+Q\right)_{\text {on }}^{-}+i o\right)} .
\end{aligned}
$$

We recall the on-minus-shell value $k_{1}^{\prime-}$ on $=\frac{\vec{k}_{1 \perp}^{2}+m_{1}^{2}}{k_{1}^{\prime+}}$ used in the above equation.

The current operator develops a singularity in the case of constant vertices for composite two-boson systems, due to the denominator $k_{1}^{\prime+}\left(Q^{+}-k_{1}^{\prime+}\right)\left(Q^{-}-k_{1 \text { on }}^{\prime-}-(Q-\right.$ $\left.\left.k_{1}^{\prime}\right)_{\text {on }}^{-}+i o\right) \sim Q^{+}$in Eq. (85), which vanishes in the limit of $Q^{+} \rightarrow 0$. In that case the term $\left(k_{1}^{+}-k_{1}^{\prime+}+Q^{+}\right)\left(K_{f}^{-}-\right.$ $\left.k_{1 \text { on }}^{\prime-}-\left(K_{i}-k_{1}\right)_{\text {on }}^{-}-\left(k_{1}-k_{1}^{\prime}+Q\right)_{\text {on }}^{-}+i o\right)$, which contains the exchanged boson, is not present. Therefore, the singularity occurring for $Q^{+} \rightarrow 0$ survives and contributes to the computation of the form factor with the bad current [12].

In the present model calculation, this problem disappears due to the one-boson exchange. The $1 / Q^{+}$singularity is canceled out by the denominator $\left[\left(k_{1}^{+}-k_{1}^{\prime+}+\right.\right.$ $\left.\left.Q^{+}\right)\left(K_{f}^{-}-k_{1 \text { on }}^{\prime-}-\left(K_{i}-k_{1}\right)_{\text {on }}^{-}-\left(k_{1}-k_{1}^{\prime}+Q\right)_{\text {on }}^{-}+i o\right)\right]^{-1}$ that goes as $\sim Q^{+}$. Therefore, in this example only the current operator represented by diagrams (a) and (e) in
Figs. 2 and 3, respectively, survives in the Drell-Yan-West frame.

\section{Current conservation and covariance}

The conservation of the matrix elements of the electromagnetic current can be obtained in the quasipotential expansion as we have shown formally and by means of the example. However, the electromagnetic (EM) form factors of a given state, calculated nonperturbatively, will be frame dependent in respect to nonkinematical boosts. Frame independence really requires full covariance, only reached exactly without truncation. Therefore, the truncated current of the system cannot transform as a relativistic four vector. Correspondingly, the continuity equation does not hold at the same level of full relativistic covariance but only up to the level of covariance under kinematical boosts.

In our test case, for example, one could get the elastic form factor of a bound state in a scalar channel by either 
computing the matrix element of the plus or the minus component of the current operator in the Breit frame with $Q^{+}=0$. The resulting form factor will depend on the component evaluated as the quasipotential expansion truncates the Fock space and full covariance is lost. (Note that the transformation of $z$ to $-z$, that exchanges plus and minus Lorentz components, is nonkinematical.) Obviously, as the quasipotential expansion is carried on such effects are minimized, as already seen in the computation of the masses of bound states for the bosonic model [6,23]. For strongly bound scalar systems in the Wick-Cutkosky model, it was also shown numerically that the inclusion of higher Fock components are necessary to saturate the covariant normalization condition [25].

We should not only focus the discussion in scalar composite systems. Another relevant aspect in the study of the EM current operator is its application to calculate form factors of higher angular momentum states of interacting two-boson systems. Then, immediately, a question arises about the fulfillment of the angular condition by the matrix elements of the plus component of the current operator for composite vector bosons. There is a rich literature discussing the subject [28]. The angular condition, in the Breit frame with $Q^{+}=0$, comes along with the constraint of rotational invariance around the momentum transfer direction. A rotation around the transverse direction is a nonkinematical boost in light-front dynamics, which has nondiagonal matrix elements in the Fock space. The symmetry associated with this particular rotation is realized precisely only when the Fock space is not truncated.

However, in the example, the matrix elements of the plus, minus, and transverse components of the truncated current operator if evaluated between composite spin 1 states will satisfy current conservation, since the valence wave function is a solution of the corresponding truncated Bethe-Salpeter equation. Therefore, the resulting expression of current conservation gives a relation between matrix elements of different Lorentz components of the current. This does not imply the full covariance of the calculation. The plus component of the current in the Breit frame with $Q^{+}=0$ has four independent matrix elements, consistent with kinematical boost invariance properties which are kept by the quasipotential expansion at any given finite order. These matrix elements satisfy the angular condition exactly only when the quasipotential expansion is taken to infinite order, or when the Fock space is not truncated.

In contrast, covariance and current conservation are fulfilled simultaneously, in a light-front perturbative calculation of the photon absorption amplitude with oneboson-exchange interaction, where the initial and final states correspond to free particles with on-minus-shell momentum. In this calculation we consider the diagrams of Fig. 2 plus (a), (c), and (d), with the initial and final states reversed.
Therefore, it is necessary to distinguish between perturbative and nonperturbative calculation of the initial and final wave functions with the truncated effective potential. In the last case, the electromagnetic properties will be frame dependent considering nonkinematical boosts, while the perturbative light-front calculation is fully covariant. In practice, it is hoped that few terms in the quasipotential expansion suffice to put under a reasonable control the effects of covariance violation as has been shown in the calculation of the bound-state mass [6,21-24]).

\section{CONCLUSION}

Our work proposes a conserved electromagnetic current operator in the quasipotential approach to the light-front dynamics for an interacting two-boson system in finite mass boson exchange models. The current operator can be perturbatively calculated in the quasipotential expansion or according to the number of particles exchanged at a given light-front time. The explicit form of the truncated and conserved light-front current has been derived. Its divergence has been shown to satisfy a Ward-Takahashi identity with the truncated three-dimensional light-front interaction.

The technical procedure is developed for a two-boson case and the construction of the light-front current is straightforward allowing one to go beyond the ladder approximation, consistently with the corresponding solution of the light-front Bethe-Salpeter equation. The relation between the light-front reduction of the Bethe-Salpeter amplitudes and current and the 4-dimensional counterpart was discussed in detail for bound and scattering states. In particular, we have shown that the matrix elements of the 4-dimensional current operator can be recovered from the corresponding light-front ones.

We observe that in general the light-front quantities do not display explicit covariance, even without any truncation, but we point out that a full covariant description is restored in the final step of the calculation, i.e., in the evaluation of matrix elements of observable operators without truncating the quasipotential expansion. This is simply stated by the equality between the matrix elements of the three- and four-dimensional current operators. Furthermore, the suggested truncation of the conserved light-front electromagnetic current operator violates covariance, even for the corresponding BS quantities. The lack of covariance can be reduced at any desired accuracy by increasing the order $n$ of the truncated quasipotential, meaning that higher intermediate Fock states should be considered in the computation of the two-body effective interaction. Indeed, the expansion in the Fock space is rapidly converging for a given covariant model (see [6,21-24]). Therefore, in practice only a few terms in the quasipotential expansion are needed to put the effects of covariance violation under reasonable control. 
We have explored in one example of the ladder BetheSalpeter equation, how our formal procedure is realized in practice. We computed all Lorentz components of the current operator in the lowest nontrivial order of the quasipotential expansion to single out the Ward-Takahashi identity. The good, transverse, and bad components of the current were derived. In non-Drell-Yan-West frames, the pair contribution is necessary for current conservation. Moreover, the pair contribution to the bad current operator due to the one-boson-exchange has no end-point singularity in the limit of $Q^{+} \rightarrow 0$, differently to models that have pointlike vertices. Although, the one-boson-exchange model reduces to the model with pointlike vertices when the mass of the exchanged boson goes to infinity with a fixed ratio $g_{S} / \mu$, the difficulty still remains because this limit does not commute with $Q^{+} \rightarrow 0$ in the computation of the form factor from the bad component of the current. We remark that the end-point singularity contributes to the bad component of the current with pointlike vertices in spin 0 composite bosonic systems [12].

We pointed out that the truncated current of the system does not transform as the relativistic four vector. Therefore, current conservation does not stand at the same level of full relativistic covariance but only up to the level of covariance under kinematical light-front boosts. Full relativistic covariance is lost because the matrix elements of the proposed current operator are taken between two-boson valence states calculated nonperturbatively with the corresponding truncated effective interaction. Although, the matrix elements of the current satisfy current conservation, the electromagnetic properties will be frame dependent in respect to nonkinematical boosts.

Our approach is complementary to Kvinikhidze and Blankleider gauging techniques [8], and it is possible to apply it beyond the bosonic model. The concepts needed for an extension to fermions were already developed in Ref. [7], but important technical details for the charge and current operators have not yet been worked out. For example, instantaneous terms from the fermion propagators ought to be carefully treated to arrive to a consistent definition of these operators. The relevance of the instantaneous terms has also been pointed out recently [27] in the calculation of the box-diagram in the Yukawa theory.

Although the paper has not discussed the relationship of the theoretical framework with real systems, we believe that it offers the route to describe light-front wave functions and consistent electromagnetic current operators for two-particle systems in finite mass boson exchange models, which can be used to improve our understanding and to control approximations performed in phenomenological applications.

\section{ACKNOWLEDGMENTS}

We thank E. Pace and G. Salmè for their critical reading of the manuscript and valuable comments. The work was supported by a Brazilian graduate-student grant (J. A. O. M.) from FAPESP and by research grants (T.F.) from CNPq and FAPESP. P. U.S. thanks the hospitality at Instituto Tecnológico de Aeronáutica.

\section{APPENDIX A: GLOBAL LIGHT-FRONT TIME PROPAGATOR}

The free two-boson Green's function in light-front coordinates allowing individual $x^{+}$-time propagation reads

$$
\begin{aligned}
\left\langle x_{1}^{\prime+} x_{2}^{\prime+}\left|G_{0}\right| x_{1}^{+} x_{2}^{+}\right\rangle= & -\frac{1}{(2 \pi)^{2}} \int d k_{1}^{-} d K^{-} e^{-(i / 2) k_{1}^{-}\left(x_{1}^{\prime+}-x_{2}^{\prime+}-x_{1}^{+}+x_{2}^{+}\right)} e^{-(i / 2) K^{-}\left(x_{2}^{\prime+}-x_{2}^{+}\right)} \\
& \times \frac{1}{\hat{k}_{1}^{+}\left(K^{+}-\hat{k}_{1}^{+}\right)\left(k_{1}^{-}-\frac{\hat{\hat{k}}_{1 \perp}^{2}+m_{1}^{2}-i o}{\hat{k}_{1}^{+}}\right)\left(K^{-}-k_{1}^{-}-\frac{\hat{\hat{k}}_{2 \perp}^{2}+m_{2}^{2}-i o}{K^{+}-\hat{k}_{1}^{+}}\right)},
\end{aligned}
$$

where the light-front momentum are $k_{i}=\left(k_{i}^{-}:=k^{0}-k^{3}, k_{i}^{+}:=k^{0}+k^{3}, \vec{k}_{\perp}\right)$. The free propagation between the hyperplanes $x_{1}^{+}=x_{2}^{+}=x^{+}$and $x_{1}^{\prime+}=x_{2}^{\prime+}=x^{\prime+}$ reduces to

$$
\begin{aligned}
\left\langle x^{\prime+} x^{\prime+}\left|G_{0}\right| x^{+} x^{+}\right\rangle & =\int \frac{d K^{-}}{2 \pi} e^{-(i / 2) K^{-}\left(x^{++}-x^{+}\right)} \int d k_{1}^{\prime-} d k_{1}^{-}\left\langle k_{1}^{\prime-}\left|G_{0}(K)\right| k_{1}^{-}\right\rangle \\
& \equiv \int \frac{d K^{-}}{2 \pi} e^{-(i / 2) K^{-}\left(x^{\prime+}-x^{+}\right)}\left|G_{0}(K)\right|
\end{aligned}
$$

where the relative $x^{+}$-time between the particles was eliminated in favor of a global time. We introduce in Eq. (A2) the notation 


$$
\left\langle k_{1}^{\prime-}\left|G_{0}(K)\right| k_{1}^{-}\right\rangle=-\frac{1}{2 \pi} \frac{\delta\left(k_{1}^{\prime-}-k_{1}^{-}\right)}{\hat{k}_{1}^{+}\left(K^{+}-\hat{k}_{1}^{+}\right)\left(k_{1}^{-}-\frac{\hat{\hat{k}}_{1 \perp}^{2}+m_{1}^{2}-i o}{\hat{k}_{1}^{+}}\right)\left(K^{-}-k_{1}^{-}-\frac{\hat{\hat{k}}_{2 \perp}^{2}+m_{2}^{2}-i o}{K^{+}-\hat{k}_{1}^{+}}\right)} .
$$

The two $k^{-}$integrations in Eq. (A2) on the bra- and ketstates are denoted by a "bar" on the left and right, respectively, with the following notation:

$$
\begin{aligned}
\left|G_{0}(K)\right| & :=\int d k_{1}^{\prime-} d k_{1}^{-}\left\langle k_{1}^{\prime-}\left|G_{0}(K)\right| k_{1}^{-}\right\rangle \\
& =\frac{i \theta\left(K^{+}-\hat{k}_{1}^{+}\right) \theta\left(\hat{k}_{1}^{+}\right)}{\hat{k}_{1}^{+}\left(K^{+}-\hat{k}_{1}^{+}\right)\left(K^{-}-\hat{k}_{1 \mathrm{on}}^{-}-\hat{k}_{2 \mathrm{on}}^{-}+i o\right)} \\
& :=g_{0}(K),
\end{aligned}
$$

where $K^{+}>0$ is chosen. The difference between $K^{+} \geq 0$ and $K^{+}>0$ comes from a contribution of the form $\delta\left(k^{+}\right) / k^{+}$related to zero modes and to the renormalization problem of the quantum field theory on the light-front [4].

The resolvent $g_{0}(K)$ is the Fourier transform of the global light-front time free propagator. It is an operator with respect to functions of the "kinematic" momentum $\left(k_{1}^{+}, \vec{k}_{1 \perp}\right), \hat{k}_{1 \text { on }}^{-}=\frac{\hat{\vec{k}}_{1 \perp}^{2}+m_{1}^{2}}{\hat{k}_{1}^{+}}$and $\hat{k}_{2}^{-}$on $=\frac{\left(\vec{K}_{\perp}-\hat{\vec{k}}_{1 \perp}\right)^{2}+m_{2}^{2}}{K^{+}-\hat{k}_{1}^{+}}$and depends parametrically on the total 4-momentum $K^{\mu}$.

The basis states for functions of the kinematical lightfront variables are defined as eigenfunctions of the momentum operators $\left(\hat{k}_{i}^{+}, \hat{\vec{k}}_{i \perp}\right)$ and the free energy operator $\hat{k}_{i \text { on }}^{-}$:

$$
\left\langle x_{i}^{-} \vec{x}_{i \perp} \mid k_{i}^{+} \vec{k}_{i \perp}\right\rangle=e^{-l\left((1 / 2) k_{i}^{+} x_{i}^{-}-\vec{k}_{i \perp} \cdot \vec{x}_{i \perp}\right)} .
$$

The states $\left|k^{+} \vec{k}_{\perp}\right\rangle$ form an orthonormal and complete basis in the space of functions of the kinematical variables, e.g.,

$$
\begin{aligned}
& \int \frac{d k^{+} d^{2} k_{\perp}}{2(2 \pi)^{3}}\left\langle x^{\prime-} \vec{x}_{\perp}^{\prime} \mid k^{+} \vec{k}_{\perp}\right\rangle\left\langle k^{+} \vec{k}_{\perp} \mid x^{-} \vec{x}_{\perp}\right\rangle \\
& =\delta\left(x^{\prime-}-x^{-}\right) \delta\left(\vec{x}_{\perp}^{\prime}-\vec{x}_{\perp}\right) .
\end{aligned}
$$

\section{APPENDIX B: BS AMPLITUDE AND LIGHT-FRONT WAVE FUNCTION}

In this Appendix, we show the reconstruction of the BS amplitudes from the light-front wave functions for bound and scattering states. The relation of the bound-state amplitude and the three-dimensional one was derived in detail in Ref. [6]. Here, we briefly review the main points of that discussion for bound states and after that we present the case of scattering states.

The bound state with 4-momentum $K_{B}, K_{B}^{2}=M_{B}^{2}$, produces a pole in the transition matrix $T(K)$ of the BSE. The light-front three-dimensional transition matrix $t(K)$ has also a pole at the same $K_{B}$, according to Eq. (19). The residue of $t(K)$ comes from the vertex function of the bound state $\left|\gamma_{B}\right\rangle$, and from Eq. (22) it is solution of the homogeneous equation

$$
\left|\gamma_{B}\right\rangle=w\left(K_{B}\right) g_{0}\left(K_{B}\right)\left|\gamma_{B}\right\rangle
$$

in agreement with (30) for the LF wave function, since $\left|\phi_{B}\right\rangle=g_{0}\left(K_{B}\right)\left|\gamma_{B}\right\rangle$.

The bound-state vertex, which comes from the residue of the 4-dimensional transition matrix at the corresponding pole, is a solution of the homogeneous equation as follows from (16) at the pole $K_{B}$,

$$
\left.\left|\Gamma_{B}\right\rangle=W\left(K_{B}\right) \tilde{G}_{0}\left(K_{B}\right)\left|\Gamma_{B}\right\rangle=W\left(K_{B}\right) G_{0}\left(K_{B}\right)|| \gamma_{B}\right\rangle,
$$

and it is reconstructed from the three-dimensional vertex as found by inspecting Eq. (21), as well as the BS amplitude

$$
\left.\left|\Psi_{B}\right\rangle=G_{0}\left(K_{B}\right) W\left(K_{B}\right) G_{0}\left(K_{B}\right)\left|g_{0}^{-1}\right| \phi_{B}\right\rangle,
$$

where the light-front wave function was introduced according to $\left|\Psi_{B}\right\rangle=G_{0}\left(K_{B}\right)\left|\Gamma_{B}\right\rangle$. Adding to Eq. (B3) $\left.G_{0}\left(K_{B}\right)\left|\left(g_{0}\left(K_{B}\right)^{-1}-w\left(K_{B}\right)\right)\right| \phi_{B}\right\rangle=0$, and making use of the expression of $w\left(K_{B}\right)$ from Eq. (23), we recover the BS amplitude for the bound state from the threedimensional wave function [6]:

$$
\left.\left|\Psi_{B}\right\rangle=\left[1+\Delta_{0}\left(K_{B}\right) W\left(K_{B}\right)\right] G_{0}\left(K_{B}\right)\left|g_{0}\left(K_{B}\right)^{-1}\right| \phi_{B}\right\rangle .
$$

The Bethe-Salpeter amplitude $\left|\Psi_{B}\right\rangle$ and its threedimensional auxiliary version $\left.\left|\phi_{B}\right\rangle=\| \Psi_{B}\right\rangle$ have to be normalized as discussed [6]. Note that the 4-dimensional normalization condition imposes that the sum over the probabilities of all Fock components of the bound state totalizes unity.

In the next, we show that the BS amplitude for the scattering state can be recovered from the threedimensional scattering wave function. To accomplish that we begin by writing the BS scattering amplitude

$$
\left|\Psi^{+}\right\rangle=\left|\Psi_{0}\right\rangle+G_{0}(K) T(K)\left|\Psi_{0}\right\rangle,
$$

as given by the solution of the scattering equation in terms of the transition matrix. Substituting the $T$-matrix integral equation in terms of the quasipotential (16) in (B5) yields

$$
\begin{aligned}
\left|\Psi^{+}\right\rangle= & \left|\Psi_{0}\right\rangle+G_{0}(K) W(K)\left|\Psi_{0}\right\rangle \\
& +G_{0}(K) W(K) G_{0}(K)\left|g_{0}(K)^{-1}\right| G_{0}(K) T(K)\left|\Psi_{0}\right\rangle \\
= & \left|\Psi_{0}\right\rangle+G_{0}(K) W(K)\left|\Psi_{0}\right\rangle \\
& +G_{0}(K) W(K) G_{0}(K)\left|g_{0}(K)^{-1}\right|\left(\left|\Psi^{+}\right\rangle-\left|\Psi_{0}\right\rangle\right) .
\end{aligned}
$$

The free 4-dimensional amplitude is related to the threedimensional one by 


$$
\left.\left|\Psi_{0}\right\rangle=G_{0}(K)\left|g_{0}(K)^{-1}\right| \phi_{0}\right\rangle .
$$

It is important to observe that the three-dimensional quantities are all evaluated with the $+i o$ prescription.

The light-front scattering state $\left|\phi^{+}\right\rangle$is the projection of the BS amplitude of the scattering state at equal $x^{+}$-times. To show the validity of this statement, it is easy to perform the bar operation on the ket-state equation (B6). The state $\left.\| \Psi^{+}\right\rangle$is a solution of the three-dimensional scattering equation (31) and consequently $\left.\| \Psi^{+}\right\rangle=\left|\phi^{+}\right\rangle$. Introducing this equality in Eq. (B6) together with (B7),

$$
\left.\left|\Psi^{+}\right\rangle=\left|\Psi_{0}\right\rangle+G_{0}(K) W(K) G_{0}(K)\left|g_{0}(K)^{-1}\right| \phi^{+}\right\rangle .
$$

Adding to (B8), the identity

$$
G_{0}(K)\left|g_{0}(K)^{-1}\left(\left|\phi^{+}\right\rangle-\left|\phi_{0}\right\rangle-g_{0}(K) w(K)\left|\phi^{+}\right\rangle\right)=0,\right.
$$

and rearranging terms one easily gets that

$$
\left.\left|\Psi^{+}\right\rangle=\left[1+\Delta_{0}(K) W(K)\right] G_{0}(K)\left|g_{0}(K)^{-1}\right| \phi^{+}\right\rangle,
$$

which is the scattering counterpart of Eq. (B4) for the bound state.

\section{APPENDIX C: REVERSE LF-TIME OPERATION}

In this Appendix we prove the identity $G \mid g^{-1}=[1+$ $\left.\Delta_{0} W\right] G_{0} \mid g_{0}^{-1}$, which reconstructs the 4-dimensional BS amplitude from the light-front wave function. (To simplify the notation the dependence on total momentum is not explicitly shown.)

The 4-dimensional Green's function, written in terms of the $T$-matrix from the quasipotential scattering equation (16) is

$$
G=G_{0}+G_{0} W G_{0}+G_{0} W \tilde{G}_{0} T G_{0},
$$

applying the bar operation on the right, i.e., integration on the bra-state, one has

$$
\begin{aligned}
G \mid & =G_{0}\left|+G_{0} W G_{0}\right|+G_{0} W G_{0}\left|g_{0}^{-1}\right| G_{0} T G_{0} \mid \\
& =G_{0}\left|+G_{0} W G_{0}\right| g_{0}^{-1} g,
\end{aligned}
$$

where the explicit form of $\tilde{G}_{0}$ and $\left|G_{0} T G_{0}\right|=g-g_{0}$ [obtained from Eq. (24)] were used. Operating on the right with the inverse of the LF Green's function, $g^{-1}=g_{0}^{-1}-$ $w$ [from Eq. (25)], on Eq. (C2), it is found that

$$
G\left|g^{-1}=G_{0}\right| g_{0}^{-1}-G_{0}\left|w+G_{0} W G_{0}\right| g_{0}^{-1},
$$

which is identical to

$$
G\left|g^{-1}=\left[1+\left(G_{0}-\tilde{G}_{0}\right) W\right] G_{0}\right| g_{0}^{-1},
$$

by using Eq. (23) for the definition of $w=$ $g_{0}^{-1}\left|G_{0} W G_{0}\right| g_{0}^{-1}$.

\section{APPENDIX D: LF CHARGE OPERATOR}

The light-front time charge operator will be derived by projecting the product of 4-dimensional operators and showing that

$$
\begin{aligned}
& \mid \hat{e} \hat{A}=\int d k^{-}\left\langle k^{-}\left|\hat{e} \hat{A}=\hat{e}_{\mathrm{LF}}\right| \hat{A},\right. \\
& \left.\hat{A} \hat{e}\left|=\int d k^{-} \hat{A} \hat{e}\right| k^{-}\right\rangle=\hat{A} \mid \hat{e}_{\mathrm{LF}}
\end{aligned}
$$

are valid.

Now we use the unity resolutions and the matrix elements of the 4-dimensional charge operator (11):

$$
\begin{aligned}
\int d k^{-}\left\langle k^{-}, k^{+}, \vec{k}_{\perp}\right| \hat{e} \hat{A}= & \int d k^{-} d^{4} p\left\langle k^{-}, k^{+}, \vec{k}_{\perp}|\hat{e}| p\right\rangle\langle p| \hat{A} \\
= & \int d k^{-} d^{4} p e \delta^{4}(k-p-Q)\langle p| \hat{A} \\
= & \int d^{4} p e \delta\left(k^{+}-p^{+}-Q^{+}\right) \\
& \times \delta^{2}\left(\vec{k}_{\perp}-\vec{p}_{\perp}-\vec{Q}_{\perp}\right)\langle p| \hat{A},
\end{aligned}
$$

from where the matrix element of the light-front charge operator is identified:

$$
\begin{aligned}
\left\langle k^{+}, \vec{k}_{\perp}\left|\hat{e}_{\mathrm{LF}}\right| p^{+}, \vec{p}_{\perp}\right\rangle:= & e \delta\left(k^{+}-p^{+}-Q^{+}\right) \\
& \times \delta^{2}\left(\vec{k}_{\perp}-\vec{p}_{\perp}-\vec{Q}_{\perp}\right) .
\end{aligned}
$$

Equation (D2) can be written in the operator form according to $\left|\hat{e} \hat{A}=\hat{e}_{\mathrm{LF}}\right| \hat{A}$ as well. Analogously, one could derive that $\hat{A} \hat{e}|=\hat{A}| \hat{e}_{\mathrm{LF}}$.

\section{APPENDIX E: CONSISTENCE OF THE LIGHT-FRONT WTI}

The purpose of this Appendix is to verify the consistence of the derivation of the light-front WTI presented in Sec. III, beginning with the EM current operator

$$
j^{\mu}=g_{0}^{-1}\left|G_{0}\left[1+W \Delta_{0}\right] \mathcal{J}^{\mu}\left[1+\Delta_{0} W\right] G_{0}\right| g_{0}^{-1},
$$

obtained in Ref. [6]. We remind that, in Sec. III, the LF electromagnetic current operator was written through the reverse LF-time operation [see Eq. (33) and Appendix C]. (To simplify the notation the dependence on total momentum is not explicitly shown.)

The divergence of the light-front EM current is

$$
Q_{\mu} j^{\mu}=g_{0}^{-1}\left|G_{0}\left(1+W \Delta_{0}\right)\left[G^{-1}, \hat{e}\right]\left(1+\Delta_{0} W\right) G_{0}\right| g_{0}^{-1},
$$

where the commutator is the divergence of the 4dimensional current operator.

Using that $G^{-1}=G_{0}^{-1}-V$ and $\Delta_{0} \hat{e} G_{0}^{-1} G_{0} \mid=$ $\Delta_{0} \mid \hat{e}_{\mathrm{LF}}=0$ [obtained from Eq. (D1)], as $\tilde{G}_{0}\left|=G_{0}\right|$, Eq. (E2) can be written as 


$$
\begin{aligned}
Q_{\mu} j^{\mu}= & g_{0}^{-1} \mid G_{0}\left(\left[G_{0}^{-1}-V, \hat{e}\right]+W \Delta_{0}\left(G_{0}^{-1} \hat{e}+\hat{e} V-V \hat{e}\right)\right. \\
& +\left(-\hat{e} G_{0}^{-1}+\hat{e} V-V \hat{e}\right) \Delta_{0} W \\
& \left.+W \Delta_{0}\left[G_{0}^{-1}-V, \hat{e}\right] \Delta_{0} W\right) G_{0} \mid g_{0}^{-1} .
\end{aligned}
$$

It is not difficult to demonstrate that

$$
\Delta_{0}\left[G_{0}^{-1}, \hat{e}\right] \Delta_{0}=\left[\hat{e}, \Delta_{0}\right] .
$$

Using this identity and the integral equation $W-V=$ $V \Delta_{0} W$ in (E3),

$$
\begin{aligned}
Q_{\mu} j^{\mu}= & g_{0}^{-1} \mid G_{0}\left(\left[G_{0}^{-1}-V, \hat{e}\right]+W \Delta_{0}\left(G_{0}^{-1} \hat{e}+\hat{e} V\right)\right. \\
& -(W-V) \hat{e}-\left(\hat{e} G_{0}^{-1}+V \hat{e}\right) \Delta_{0} W \\
& +\hat{e}(W-V)+W\left[\hat{e}, \Delta_{0}\right] W-(W-V) \hat{e} \Delta_{0} W \\
& \left.+W \Delta_{0} \hat{e}(W-V)\right) G_{0} \mid g_{0}^{-1}
\end{aligned}
$$

is obtained. After all cancellations, we get

$$
\begin{aligned}
Q_{\mu} j^{\mu}= & g_{0}^{-1} \mid G_{0}\left(\left[G_{0}^{-1}, \hat{e}\right]+W \Delta_{0} G_{0}^{-1} \hat{e}-W \hat{e}\right. \\
& \left.-\hat{e} G_{0}^{-1} \Delta_{0} W+\hat{e} W\right) G_{0} \mid g_{0}^{-1} \\
= & {\left[g_{0}^{-1}, \hat{e}_{\mathrm{LF}}\right]-g_{0}^{-1}\left|G_{0} W G_{0}\right| g_{0}^{-1}\left|\hat{e} G_{0}\right| g_{0}^{-1} } \\
& \left.+g_{0}^{-1}\left|G_{0} \hat{e}\right| g_{0}^{-1} \mid G_{0} W\right) G_{0} \mid g_{0}^{-1} .
\end{aligned}
$$

From Eq. (E7), using the property (D1),

$$
Q_{\mu} j^{\mu}=\left[g_{0}^{-1}-w, \hat{e}_{\mathrm{LF}}\right],
$$

the light-front WTI is found in agreement with (37).

\section{APPENDIX F: CONSERVED LF CURRENT AT $\mathcal{O}(n)$}

The aim of this Appendix is to derive a conserved lightfront current operator consistent with the expansion of the quasipotential in powers of $V$ at a given order. For the sake of simplicity of the expressions, in this Appendix we will not exhibit the explicit dependence on the total 4momentum of the operators.

The 4-dimensional current operator has two parts,

$$
\mathcal{J}^{\mu}=\mathcal{J}_{0}^{\mu}+\mathcal{J}_{I}^{\mu},
$$

the first one is the free current and the second term carries the interaction. For example, it may contain crossed diagrams.

The 4-dimensional current operator satisfies the WTI of Eq. (9). It corresponds to two independent identities, one for the free part and another for the interacting term

$$
\begin{gathered}
Q_{\mu} \mathcal{J}_{0}^{\mu}=\left[G_{0}^{-1}, \hat{e}\right], \\
Q_{\mu} \mathcal{J}_{I}^{\mu}=[\hat{e}, V],
\end{gathered}
$$

respectively. The current operator $\mathcal{J}_{I}^{\mu}$ is of order 1 in the interaction, as expressed by Eq. (F3).

We remind that the expansion of $W$ in terms of the potential $V$ is

$$
\begin{gathered}
W^{(n)}=\sum_{i=1}^{n} W_{i} \\
W_{i}=V\left(\Delta_{0} V\right)^{i-1} .
\end{gathered}
$$

The current at $\mathcal{O}(n)$ in the interaction is found by collecting all terms up to that given order, using that $\mathcal{J}_{I}^{\mu}$ is $\mathcal{O}(1)$ in $V$, and the expansion of $W$ from Eqs. (F4) and (F5) in the current operator of Eq. (34),

$$
\begin{aligned}
j^{c \mu(n)}= & g_{0}^{-1} \mid G_{0}\left[\mathcal{J}^{\mu}+\sum_{i=1}^{n-1}\left(W_{i} \Delta_{0} \mathcal{J}^{\mu}+\mathcal{J}^{\mu} \Delta_{0} W_{i}\right)\right. \\
& +\sum_{i=2}^{n-1} \sum_{j=1}^{i-1} W_{j} \Delta_{0} \mathcal{J}^{\mu} \Delta_{0} W_{i-j}+W_{n} \Delta_{0} \mathcal{J}_{0}^{\mu} \\
& \left.+\mathcal{J}_{0}^{\mu} \Delta_{0} W_{n}+\sum_{i=1}^{n-1} W_{i} \Delta_{0} \mathcal{J}_{0}^{\mu} \Delta_{0} W_{n-i}\right] G_{0} \mid g_{0}^{-1} .
\end{aligned}
$$

The proposed form satisfies current conservation, as we prove in the following by induction.

For order $n=0$ the current corresponds to the free one:

$$
j^{c \mu(0)}=g_{0}^{-1}\left|G_{0} \mathcal{J}_{0}^{\mu} G_{0}\right| g_{0}^{-1},
$$

and satisfies

$$
Q_{\mu} j^{c \mu(0)}=\left[g_{0}^{-1}, \hat{e}_{\mathrm{LF}}\right] .
$$

The conservation of the $n=1 \mathrm{LF}$ current is shown in the next. The $n=1$ current is

$$
j^{c \mu(1)}=g_{0}^{-1}\left|G_{0}\left[\mathcal{J}^{\mu}+W_{1} \Delta_{0} \mathcal{J}_{0}^{\mu}+\mathcal{J}_{0}^{\mu} \Delta_{0} W_{1}\right] G_{0}\right| g_{0}^{-1} .
$$

Using the 4-dimensional WTI (9), the corresponding expression for the free-current equation (F2) and that $W_{1}=$ $V$, we write

$$
\begin{aligned}
Q_{\mu} j^{c \mu(1)}= & g_{0}^{-1} \mid G_{0}\left[G_{0}^{-1} \hat{e}-\hat{e} G_{0}^{-1}-V \tilde{G}_{0} G_{0}^{-1} \hat{e}\right. \\
& -V \Delta_{0} \hat{e} G_{0}^{-1}+G_{0}^{-1} \hat{e} \Delta_{0} V \\
& \left.+\hat{e} G_{0}^{-1} \tilde{G}_{0} V\right] G_{0} \mid g_{0}^{-1} .
\end{aligned}
$$

Using

$$
\left|\Delta_{0}=\right| G_{0}-\left|G_{0}\right| g_{0}^{-1} \mid G_{0}=0,
$$

the analogous identity $\Delta_{0}|=0,| \hat{e} \Delta_{0}=\hat{e}_{\mathrm{LF}} \mid \Delta_{0}=0$, and $\Delta_{0} \hat{e}\left|=\Delta_{0}\right| \hat{e}_{\mathrm{LF}}=0$ [obtained from Eq. (D1)], one gets for Eq. (F10)

$$
\begin{aligned}
Q_{\mu} j^{c \mu(1)}= & g_{0}^{-1} \hat{e}_{\mathrm{LF}}-\hat{e}_{\mathrm{LF}} g_{0}^{-1}-g_{0}^{-1}\left|G_{0} V \tilde{G}_{0} G_{0}^{-1} \hat{e} G_{0}\right| g_{0}^{-1} \\
& +g_{0}^{-1}\left|G_{0} \hat{e} G_{0}^{-1} \tilde{G}_{0} V G_{0}\right| g_{0}^{-1} .
\end{aligned}
$$

Substituting $\tilde{G}_{0}$ from Eq. (14) and rearranging terms, the expression for the $n=1$ light-front WTI identity comes as 


$$
Q_{\mu} j^{c \mu(1)}=\left[g_{0}^{-1}, \hat{e}_{\mathrm{LF}}\right]+\left[\hat{e}_{\mathrm{LF}}, w^{(1)}\right]=\left[g_{(1)}^{-1}, \hat{e}_{\mathrm{LF}}\right]
$$

reminding that $w^{(1)}=g_{0}^{-1}\left|G_{0} V G_{0}\right| g_{0}^{-1}$.

The proof by induction of the WTI at order $n+1$ begins with the separation of the $Q_{\mu} j^{c \mu(n)}$ in the expression of the divergence of $j^{c \mu(n+1)}$,

$$
\begin{aligned}
Q_{\mu} j^{c \mu(n+1)}= & Q_{\mu} j^{c \mu(n)}+g_{0}^{-1} \mid G_{0}\left(W_{n} \Delta_{0}[\hat{e}, V]\right. \\
& +[\hat{e}, V] \Delta_{0} W_{n}+\sum_{i=1}^{n-1} W_{i} \Delta_{0}[\hat{e}, V] \Delta_{0} W_{n-i} \\
& +W_{n+1} \Delta_{0}\left[G_{0}^{-1}, \hat{e}\right]+\left[G_{0}^{-1}, \hat{e}\right] \Delta_{0} W_{n+1} \\
& \left.+\sum_{i=1}^{n} W_{i} \Delta_{0}\left[G_{0}^{-1}, \hat{e}\right] \Delta_{0} W_{n+1-i}\right) G_{0} \mid g_{0}^{-1},
\end{aligned}
$$

where we have used that $Q_{\mu} \mathcal{J}_{I}^{\mu}=[\hat{e}, V]$. Simplifying the above expression by taking into account that $\left|\Delta_{0}=\Delta_{0}\right|=$ $0, \quad \tilde{G}_{0} G_{0}^{-1} \hat{e} \Delta_{0}=\Delta_{0} \hat{e} G_{0}^{-1} \tilde{G}_{0}=0, \quad$ and $\quad W_{i} \Delta_{0} V=$ $V \Delta_{0} W_{i}=W_{i+1}$, one arrives at

$$
\begin{aligned}
Q_{\mu} j^{c \mu(n+1)}= & Q_{\mu} j^{c \mu(n)}+g_{0}^{-1} \mid G_{0}\left(W_{n} \Delta_{0} \hat{e} V-V \hat{e} \Delta_{0} W_{n}\right. \\
& +\sum_{i=1}^{n-1} W_{i} \Delta_{0} \hat{e} W_{n+1-i}-\sum_{i=1}^{n-1} W_{i+1} \hat{e} \Delta_{0} W_{n-i} \\
& -W_{n+1} \tilde{G}_{0} G_{0}^{-1} \hat{e}+\hat{e} G_{0}^{-1} \tilde{G}_{0} W_{n+1} \\
& \left.+\sum_{i=1}^{n} W_{i}\left[\hat{e}, \Delta_{0}\right] W_{n+1-i}\right) G_{0} \mid g_{0}^{-1},
\end{aligned}
$$

where we have used Eq. (E4). Canceling several terms, it remains:

$$
\begin{aligned}
Q_{\mu} j^{c \mu(n+1)}= & Q_{\mu} j^{c \mu(n)}-g_{0}^{-1} \mid G_{0}\left(W_{n+1} \tilde{G}_{0} G_{0}^{-1} \hat{e}\right. \\
& \left.-\hat{e} G_{0}^{-1} \tilde{G}_{0} W_{n+1}\right) G_{0} \mid g_{0}^{-1},
\end{aligned}
$$

and by the induction hypothesis,

$$
Q_{\mu} j^{c \mu(n+1)}=\left[g_{0}^{-1}-w^{(n)}, \hat{e}_{\mathrm{LF}}\right]-\left[w_{n+1}, \hat{e}_{\mathrm{LF}}\right],
$$

where we have recalled that $w_{n+1}=g_{0}^{-1}\left|G_{0} W_{n+1} G_{0}\right| g_{0}^{-1}$. The light-front interaction at order $n+1$ is $w^{(n+1)}=$ $w^{(n)}+w_{n+1}$; therefore,

$$
Q_{\mu} j^{c \mu(n+1)}=\left[g_{0}^{-1}-w^{(n+1)}, \hat{e}_{\mathrm{LF}}\right],
$$

completing the inductive proof of the light-front WTI for the truncated current.

\section{APPENDIX G: CORRECTION TO THE NONCONSERVED CURRENT $\Delta \boldsymbol{j}_{\mu}^{(n)}$}

The difference between the conserved and nonconserved truncated currents is derived in the following. (To simplify the notation the dependence on total momentum is not explicitly shown.)

The nonconserved EM current in $\mathcal{O}(n)$ is given by Eq. (44):

$j^{\mu(n)}=g_{0}^{-1}\left|G_{0}\left(1+\sum_{i=1}^{n} W_{i} \Delta_{0}\right) \mathcal{J}^{\mu}\left(1+\Delta_{0} \sum_{i=1}^{n} W_{i}\right) G_{0}\right| g_{0}^{-1}$

Subtracting from the expression of the truncated conserved EM current (F6), the nonconserved current (G1), the difference is written as

$$
\begin{aligned}
\Delta j^{\mu(n)}= & j^{\mu c(n)}-j^{\mu(n)} \\
= & g_{0}^{-1} \mid G_{0}\left[\sum_{i=2}^{n-1} \sum_{j=1}^{i-1} W_{j} \Delta_{0} \mathcal{J}^{\mu} \Delta_{0} W_{i-j}\right. \\
& +\sum_{i=1}^{n-1} W_{i} \Delta_{0} \mathcal{J}_{0}^{\mu} \Delta_{0} W_{n-i}-W_{n} \Delta_{0} \mathcal{J}_{I}^{\mu} \\
& \left.-\mathcal{J}_{I}^{\mu} \Delta_{0} W_{n}-\sum_{i=1}^{n} W_{i} \Delta_{0} \mathcal{J}^{\mu} \Delta_{0} \sum_{j=1}^{n} W_{j}\right] G_{0} \mid g_{0}^{-1} .
\end{aligned}
$$

Using the formulas for subtracting terms in a series,

$$
\sum_{i=1}^{n} \sum_{j=1}^{n} a_{i, j}-\sum_{i=2}^{n} \sum_{j=1}^{i-1} a_{j, i-j}=\sum_{i=1}^{n} \sum_{j=n+1-i}^{n} a_{i, j}
$$

the difference in Eq. (G2) can be presented as

$$
\begin{aligned}
\Delta j^{\mu(n)}= & -g_{0}^{-1} \mid G_{0}\left[W_{n} \Delta_{0} \mathcal{J}_{I}^{\mu}+\mathcal{J}_{I}^{\mu} \Delta_{0} W_{n}\right. \\
& +\sum_{i=1}^{n-1} W_{i} \Delta_{0} \mathcal{J}_{I}^{\mu} \Delta_{0} W_{n-i} \\
& \left.+\sum_{i=1}^{n} \sum_{j=n+1-i}^{n} W_{i} \Delta_{0} \mathcal{J}^{\mu} \Delta_{0} W_{j}\right] G_{0} \mid g_{0}^{-1}
\end{aligned}
$$


[1] P. A. M. Dirac, Rev. Mod. Phys. 21, 392 (1949).

[2] E. E. Salpeter and H. A. Bethe, Phys. Rev. 84, 1232 (1951).

[3] C. Itzykson and J.-B. Zuber, Quantum Field Theory (McGraw-Hill, Singapore, 1985).

[4] S. J. Brodsky, H.-C. Pauli, and S. S. Pinsky, Phys. Rep. 301, 299 (1998).

[5] R. M. Woloshyn and A. D. Jackson, Nucl. Phys. B64, 269 (1973).

[6] J. H. O. Sales, T. Frederico, B. V. Carlson, and P. U. Sauer, Phys. Rev. C 61, 044003 (2000).

[7] J. H. O. Sales, T. Frederico, B. V. Carlson, and P. U. Sauer, Phys. Rev. C 63, 064003 (2001).

[8] A. N. Kvinikhidze and B. Blankleider, Phys. Rev. D 68, 025021 (2003).

[9] H. W. L. Naus, J.P.C. de Melo, and T. Frederico, FewBody Syst. 24, 99 (1998).

[10] B. L. G. Bakker, H.-M. Choi, and C.-R. Ji, Phys. Rev. D 63, 074014 (2001); 65, 116001 (2002); B. L. G. Bakker and C.-R. Ji, Phys. Rev. D 71, 053005 (2005).

[11] J. P. B. C. de Melo, T. Frederico, E. Pace, and G. Salmè, Nucl. Phys. A707, 399 (2002); Braz. J. Phys. 33, 301 (2003).

[12] J.P. B. C de Melo, J.H. O. Sales, T. Frederico, and P. U. Sauer, Nucl. Phys. A631, 574C (1998).

[13] F. M. Lev, E. Pace, and G. Salmè, Nucl. Phys. A641, 229 (1998); Few-Body Syst. Suppl. 10, 135 (1999); Phys. Rev. Lett. 83, 5250 (1999); Phys. Rev. C 62, 064004 (2000); Nucl. Phys. A663, 365 (2000); E. Pace and G. Salmè, Nucl. Phys. A684, 487 (2001); A689, 441 (2001).

[14] F. Gross and D. O. Riska, Phys. Rev. C 36, 1928 (1987).

[15] A. N. Kvinikhidze and B. Blankleider, Phys. Rev. C 60, 044003 (1999); 60, 044004 (1999).

[16] B. C. Tiburzi and G. A. Miller, Phys. Rev. D 67, 054014 (2003); 67, 054015 (2003).

[17] C.-R. Ji and H.-M. Choi, Phys. Lett. B 513, 330 (2001).
[18] H.-M. Choi and C.-R. Ji, Nucl. Phys. A679, 735 (2001).

[19] J.P. B. C. de Melo, T. Frederico, E. Pace, and G. Salmè, Phys. Lett. B 581, 75 (2004).

[20] J.P. B. C. de Melo, T. Frederico, E. Pace, and G. Salmè, Phys. Rev. D 73, 074013 (2006).

[21] P. G. Blunden, M. Burkardt, and G. A. Miller, Phys. Rev. C 59, R2998 (1999); 60, 055211 (1999).

[22] G. A. Miller and R. Machleidt, Phys. Lett. B 455, 19 (1999); Phys. Rev. C60, 035202 (1999).

[23] J. R. Cooke, G. A. Miller, and D. R. Phillips, Phys. Rev. C 61, 064005 (2000).

[24] J. Carbonell and V. A. Karmanov, Eur. Phys. J. A 27, 11 (2006); V. A. Karmanov and J. Carbonell, Eur. Phys. J. A27, 1 (2006).

[25] D. S. Hwang and V. A. Karmanov, Nucl. Phys. B696, 413 (2004); V. A. Karmanov and D. S. Hwang, Few-Body Syst. 36, 155 (2005).

[26] M. Mangin-Brinet, J. Carbonell, and V.A. Karmanov, Phys. Rev. D 64, 125005 (2001); Phys. Rev. C 68, 055203 (2003).

[27] B. L. G. Bakker, J. K. Boomsma, and C.-R. Ji, Phys. Rev. D 75, 065010 (2007).

[28] L. L. Frankfurt and M. I. Strikman, Nucl. Phys. B148, 107 (1979); Phys. Rep. 76, 215 (1981); I. L. Grach and L. A. Kondratyuk, Sov. J. Nucl. Phys. 39, 198 (1984); L. L. Frankfurt, I. L. Grach, L. A. Kondratyuk, and M. Strikman, Phys. Rev. Lett. 62, 387 (1989); P. L. Chung, F. Coester, B. D. Keister, and W. N. Polyzou, Phys. Rev. C 37, 2000 (1988); B. D. Keister and W. N. Polyzou, Adv. Nucl. Phys. 20, 225 (1991); S. J. Brodsky and J. R. Hiller, Phys. Rev. D 46, 2141 (1992); L. L. Frankfurt, T. Frederico, and M.I. Strikman, Phys. Rev. C 48, 2182 (1993); B. D. Keister, Phys. Rev. D 49, 1500 (1994). For a recent discussion on the angular condition for general spin systems, see C. E. Carlson and C.-R. Ji, Phys. Rev. D 67, 116002 (2003). 